\title{
Efficacy and safety of cyclosporine A in the treatment of idiopathic membranous nephropathy in an Asian population
}

This article was published in the following Dove Press journal:

Drug Design, Development and Therapy

\author{
Shujun Lin' \\ Hong-Yan $\mathrm{Li}^{2}$ \\ Tianbiao Zhou' \\ Wenshan Lin' \\ 'Department of Nephrology, The Second \\ Affiliated Hospital of Shantou University \\ Medical College, Shantou 5I504I, \\ People's Republic of China; ${ }^{2}$ Department \\ of Nephrology, Huadu District People's \\ Hospital of Guangzhou, Southern Medical \\ University, Guangzhou 510800, People's \\ Republic of China
}

Correspondence: Tianbiao Zhou Department of Nephrology, the Second Affiliated Hospital, Shantou University Medical College, No. 69 Dongsha Road, Shantou 5I504I, People's Republic of China

Email zhoutb@aliyun.com
Introduction: The efficacy of cyclosporine A (CsA) in the treatment of idiopathic membranous nephropathy (IMN) is unclear. This meta-analysis was conducted to assess the efficacy and the safety of CsA in the treatment of IMN in Asians.

Methods: We searched the Pubmed, China Biomedical Database, CNKI, Wanfang Data, VIP, and EMBASE (November 30, 2018) systematically to identify the appropriate randomized controlled trials (RCTs) reporting the efficacy and the safety of CsA and glucocorticoid (GC) treatment vs other immunosuppressants and GC on patients with IMN in Asian populations.

Results: The CsA treated group entered complete remission (CR) faster (3 months) than a cyclophosphamide (CTX) group. While the CsA group lower inefficacy rates and higher total remission (TR, CR, or partial remission) than the CTX group in the total treatment (3 months, 6 months, and 12 months), it had a higher relapse rate. As for the CsA group vs the tacrolimus (TAC) group, the TAC had a significant effect in increasing the CR and the $\mathrm{TR}$, with decreased no remission. With the therapeutic regimens of $\mathrm{Cs}+\mathrm{GC}$ vs $\mathrm{CTX}+\mathrm{GC}$, the CsA exhibited better efficacy in lowering the proteinuria levels only at 12 months, not at 3 months or 6 months. Severe events like leucopenia, hemorrhagic cystitis, and alopecia were observed in the CTX group. Gingival hyperplasia, hirsutism, and elevated blood pressure were reported only in the CsA group. Gastrointestinal syndrome, liver function lesion, happened more frequently in the CTX group, and elevated uric acid was more common in the CsA group.

Conclusions: In brief, the CsA has better efficacy than the CTX group in the Asian population, with mild adverse effects but higher relapse rates in short-term treatment.

Keywords: idiopathic membranous nephropathy, cyclosporine A, cyclophosphamide, mycophenolate mofetil, tacrolimus

\section{Introduction}

Membranous nephropathy (MN) is the most common pathology in adults with nephrotic syndrome. Idiopathic membranous nephropathy (IMN), which consists of more than $20 \%$ of $\mathrm{MN}$, is diagnosed when patients have primary $\mathrm{MN}$ without a secondary cause such as hepatitis B related nephritis, lupus nephritis, or malignancy, etc. ${ }^{1,2}$

Whether to treat IMN has been in debate for decades due to the observation that more than $20 \%$ of the patients can achieve spontaneous remission without immunosuppressive treatments. ${ }^{3-5}$ These phenomena are more frequent in cases with lower baseline proteinuria. Some untreated IMN patients, especially young patients $(<50$ years old) with proteinuria $<4 \mathrm{~g} / \mathrm{L}$, stable renal function at 6 months and 
without the nephrotic syndrome, tend to have a good prognosis. However, some studies report that over $40 \%$ of the untreated IMN will develop into end-stage renal disease, especially in old patients with persistent proteinuria $>8 \mathrm{~g} / \mathrm{d}$ and the deteriorating renal function. ${ }^{2-4,6-8}$

Recently, when treating patients with significant kidney damage or a conservative treatment history, the Italian Ponticelli recommends treatment of IMN, suggesting the combination of the methylprednisolone with the oral cyclophosphamide (CTX). ${ }^{9}$ However, CTX leads to severe side effects in many patients, such as gonadoinhibition, marrow toxicity, hemorrhagic cystitis, hepatotoxicity, and cancer. $^{10,11}$

Cyclosporin A (CsA) is composed of a cyclic polypeptide consisting of 11 amino acids, and it is one of the most effective immunosuppressive agents. Clinically, it is mainly used for anti-rejection reaction in kidney, liver, and heart transplantation. It can also be used together with the adrenocortical hormone to treat various immune diseases. As a calcineurin inhibitor, CsA becomes active by integrating with cyclophilin. The CsA-cyclophilin will combine with calcineurin as a competitive inhibition and affect the activity of calcineurin. In this way, the CsA suppresses the production of IL-2, IL-3, and IFN- $\gamma$, as well as translocation of nuclear factor-activating $\mathrm{T}$ cells. This subsequently dampens T-cell activity, impairs the function of T-lymphocyte helpers, and decreases lymphocyte proliferation. CsA treatment increases the production of vasoconstrictor factors such as the endothelin and thromboxane, which leads to the constriction of blood vessels, decreased filtration rate, and changes in glomerular permeability. ${ }^{12-14}$ This mechanism pertains to its antiproteinuric effect and toxicity. ${ }^{15,16}$ Thus, the current meta-analysis was conducted to assess whether CsA is an effective and safe option for IMN compared with other immunosuppressive agents.

\section{Materials and methods}

\section{Search strategy}

Pubmed, China Biomedical Database, CNKI, Wanfang Data, VIP, and EMBASE were searched using the following keywords: cyclosporine, cyclosporine A, CsA, membranous nephropathy, primary membranous nephropathy, idiopathic membranous nephropathy, $\mathrm{MN}$, and IMN to select appropriate articles. The search extended to November 30, 2018.

\section{Inclusion and exclusion criteria}

\section{Inclusion criteria}

The inclusion criteria for the study were as follows: (1) study type: various kinds of randomized controlled trials (RCTs) including prospective, single-blind, or multicenter clinical trials. (2) Patients: all patients were diagnosed with IMN by renal biopsy. Only patients $\geq 18$ years old were included. (3) Drug interventions: different medication comparison, all studies including the application of CsA. (4) Studies in the Asian population.

\section{Exclusion criteria}

Exclusion criteria: (1) patients with secondary MN such as lupus nephritis, hepatitis B virus-associated nephritis, and malignancies, etc. (2) Studies that only included CsA without a control group. (3) The diagnostic criteria and detailed scheme were not clear. (4) Either the experimental group or the control group had less than 10 cases. (5) Studies with unclear data.

\section{Outcome measures}

Complete remission (CR), partial remission, invalid remission, total remission (TR; CR or partial remission), serum albumin $(\mathrm{g} / \mathrm{L}), 24 \mathrm{hr}$-proteinuria levels $(\mathrm{g})$, serum creatinine $(\mu \mathrm{mol} / \mathrm{L})$, serum triacylglycerol $(\mathrm{nmol} / \mathrm{L})$, serum cholesterol $(\mathrm{mmol} / \mathrm{L})$, white blood cell counts and adverse events were used as outcome measures.

\section{Data collection}

Two researchers screened the literature (either to read the titles and abstracts or contents) to find appropriate passages according to the designed inclusion criteria and exclusion criteria. At the same time, we organized the group discussion to deal with debatable articles. In this way, we filtered irrelevant studies.

\section{Data analysis and statistical method}

To compare and analyze the efficacy and the safety of CsA, we used Review Manager Version 5.3 software to calculate clinical indicators and adverse events of patients with diverse medication treatments. A random effects model was applied to assess data when the $P$-value of the heterogeneity test was $<0.1$. Other data were assessed by a fixed model. The weighted mean differences were used to pool the continuous data, and binary data were expressed using the odds ratio (OR). 95\% confidence intervals (CIs) were also calculated. $\chi^{2}$-test of heterogeneity $<0.05$ indicated a significant difference. 


\section{Results}

\section{Search results}

This meta-analysis included a total of 22 randomized and comparative studies ${ }^{17-38}$ in Asian populations (involving 1,366 patients, with CsA and IMN (Table 1). Sixteen studies $^{17-23,25-30,32-34}$ reported effects of CsA plus glucocorticoid (GC) vs CTX plus GC. Three studies ${ }^{24,31,35}$ reported comparisons among CsA plus GC, CTX plus GC, and mycophenolate mofetil (MMF) plus GC. Three studies ${ }^{36-38}$ compared tacrolimus (TAC) with the CsA.

\section{CR (complete remission)}

Seventeen studies ${ }^{17-28,30-34}$ assessed the effects of CsA $+\mathrm{GC}$ vs $\mathrm{CTX}+\mathrm{GC}$ on IMN, and the $\mathrm{CR}$ rate was calculated. The $\mathrm{CR}$ rate in the CsA group was significantly higher when compared with that in the CTX group at 3 months $(\mathrm{OR}=2.07,95 \% \mathrm{CI}: 1.30$ 3.29; $P=0.002$; Figure 1 and Table 2). ${ }^{17,19,23,25-28,33,34}$ While at 6 months, ${ }^{17,19,23,26-28,30,33}$ (OR $=1.46$, 95\% CI:0.99-2.16, $P=0.06$; Figure 1 and Table 2) and 12 months ${ }^{17-24,27,28,32-34}$ $(\mathrm{OR}=1.18,95 \% \mathrm{CI}: 0.90-1.55, P=0.22$; Figure 1 and Table 2$)$, this advantage was not present.

Three studies ${ }^{24,31,35}$ assessed the $\mathrm{CR}$ rate of $\mathrm{CsA}+\mathrm{GC}$ vs $\mathrm{MMF}+\mathrm{GC}$. The CsA group had similar efficacy to the MMF group $\quad(\mathrm{OR}=0.92,95 \% \mathrm{CI}: \quad 0.41-2.05, \quad P=0.83$; Table 2).

Another three studies ${ }^{24,36-38}$ compared $\mathrm{CsA}+\mathrm{GC}$ with the TAC+GC. The CsA group had lower $\mathrm{CR}$ rate when compared with the TAC group $(\mathrm{OR}=0.42,95 \% \mathrm{CI}$ : $0.21-$ $0.85, P=0.02$; Table 2).

\section{NR (no remission)}

Seventeen studies ${ }^{17-28,30-34}$ were included in the comparison of $\mathrm{CsA}+\mathrm{GC}$ vs $\mathrm{CTX}+\mathrm{GC}$. The $\mathrm{NR}$ rate of the CsA $+\mathrm{GC}$ group was less than the $\mathrm{CTX}+\mathrm{GC}$ group at 3 months ${ }^{17,19,23,25-28,33,34} \quad(\mathrm{OR}=0.52, \quad 95 \% \mathrm{CI}: \quad 0.36$ 0.74, $P=0.0004$; Table 2), 6 months ${ }^{17,19,23,26-28,30,33}$ $(\mathrm{OR}=0.62$, 95\% CI:0.41-0.93, $P=0.02$; Table 2) and 12 months ${ }^{17-24,27,28,32-34} \quad(\mathrm{OR}=0.51, \quad 95 \% \quad \mathrm{CI}: 0.36-0.73$, $P=0.0002$; Table 2).

Three studies ${ }^{24,31,35}$ compared $\mathrm{CsA}+\mathrm{GC}$ vs $\mathrm{MMF}+\mathrm{GC}$. The NR rate of the MMF group did not differ from the CsA group $\quad(\mathrm{OR}=0.55, \quad 95 \% \mathrm{CI}: 0.22-1.36, \quad P=0.20$; Table 2).

Three studies ${ }^{24,36-38}$ calculated the NR rate of CsA $+\mathrm{GC}$ vs TAC $+\mathrm{GC}$. Compared with the CsA group, TAC presented a decreased NR rate $(\mathrm{OR}=3.27,95 \% \mathrm{CI}: 1.40$ 7.64, $P=0.006$; Table 2).

\section{TR (total remission)}

Seventeen studies ${ }^{17-28,30-34}$ compared CsA+GC vs CTX + GC. TR rate at the 3 time-points suggested diverse outcomes. The CsA group had a higher rate of TR at all 3 timepoints, including 3 months ${ }^{17,19,23,25-28,33,34} \quad(P=0.0004$, $\mathrm{OR}=1.94$, 95\% CI:1.35-2.80; Figure 2 and Table 2), 6 months ${ }^{17,19,23,26-28,30,33} \quad(p=0.02, \quad \mathrm{OR}=1.62,95 \%$ CI:1.072.46; Figure 2 and Table 2) and 12 months ${ }^{17-24,27,28,32-34}$ $(P=0.0006, \mathrm{OR}=1.81,95 \% \mathrm{CI}: 1.29-2.53$; Figure 2 and Table 2).

Three studies ${ }^{24,31,35}$ compared $\mathrm{CsA}+\mathrm{GC}$ vs $\mathrm{MMF}+\mathrm{GC}$. Compared with the MMF group, we found no significant difference in the TR rate of the CsA group $(P=0.20$, $\mathrm{OR}=1.82$, 95\% CI: 0.73-4.49; Table 2).

Three included studies ${ }^{24,36-38}$ compared the TR rate of TAC $+\mathrm{GC}$ with $\mathrm{CsA}+\mathrm{GC}$. The CsA group was less effective than the TAC group $(P=0.006, \mathrm{OR}=0.31,95 \%$ CI:0.13-0.71; Table 2).

\section{Relapse rate}

Ten studies ${ }^{17-23,28,30,33}$ compared relapse rates in $\mathrm{CsA}+\mathrm{GC}$ vs $\mathrm{CTX}+\mathrm{GC}$. A higher relapse rate was observed in the $\mathrm{CsA}+\mathrm{GC}$ group compared to the $\mathrm{CTX}+\mathrm{GC}$ group $(P<0.0001, \mathrm{OR}=3.06,95 \% \mathrm{CI}: 1.84-5.07$; Table 2).

\section{Proteinuria}

In comparing $\mathrm{CsA}+\mathrm{GC}$ vs $\mathrm{CTX}+\mathrm{GC}, 11$ studies concluded that IMN patients with $\mathrm{CsA}$ treatment presented with decreased proteinuria at 12 months ${ }^{17,19-21,27,28,32,34}$ $(P=0.02)$, with a pooled mean difference of $-0.66(95 \% \mathrm{CI}$ : $-1.24,-0.08$; Figure 3 and Table 2 ), but no significant difference at 3 months $^{17,19,25-28}(P=0.11, \mathrm{OR}=-0.35,95 \% \mathrm{CI}$ : $-0.78,0.08$; Figure 3 and Table 2), or 6 months ${ }^{17,19,26-28,30}$ $(P=0.96, \mathrm{OR}=-0.01,95 \% \mathrm{CI}:-0.41$ to 0.39 ; Figure 3 and Table 2).

Two studies ${ }^{36,37}$ comparing $\mathrm{CsA}+\mathrm{GC}$ with $\mathrm{TAC}+\mathrm{GC}$ reported no differences in proteinuria $(P=0.06, \mathrm{OR}=0.36$, 95\% CI: -0.01 to 0.73 ; Table 2 ).

\section{Serum albumin}

In comparing $\mathrm{CsA}+\mathrm{GC}$ vs $\mathrm{CTX}+\mathrm{GC},{ }^{17,19,25-28}$ there were no differences in serum albumin at 3 months $(P=0.48$, $\mathrm{OR}=0.16$, 95\%CI:-0.27,0.59; Figure 4 and Table 2).

At 6 months ${ }^{17,19,26-28,30}$ and 12 months, ${ }^{17,19-21,27,28,32,34}$ the CsA group exhibited higher serum albumin levels ( $P=0.002$ at 6 months, $P=0.03$ at 12 months; Figure 4 and Table 2$)$. The pooled mean differences were $0.56(95 \% \mathrm{CI}$ : $0.21,0.90)$ and 1.40 (95\% CI: $0.15,2.64)$, respectively. 


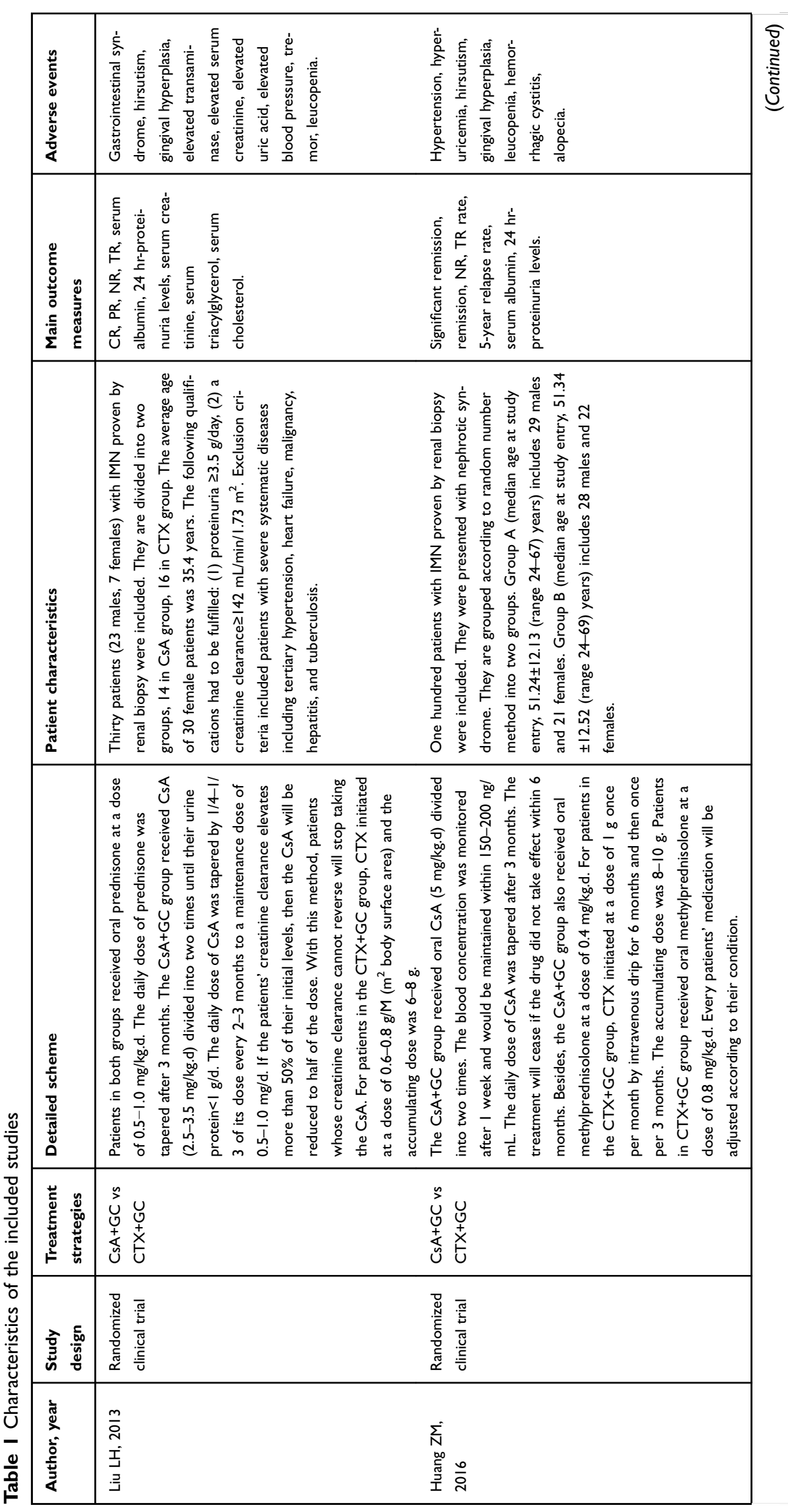




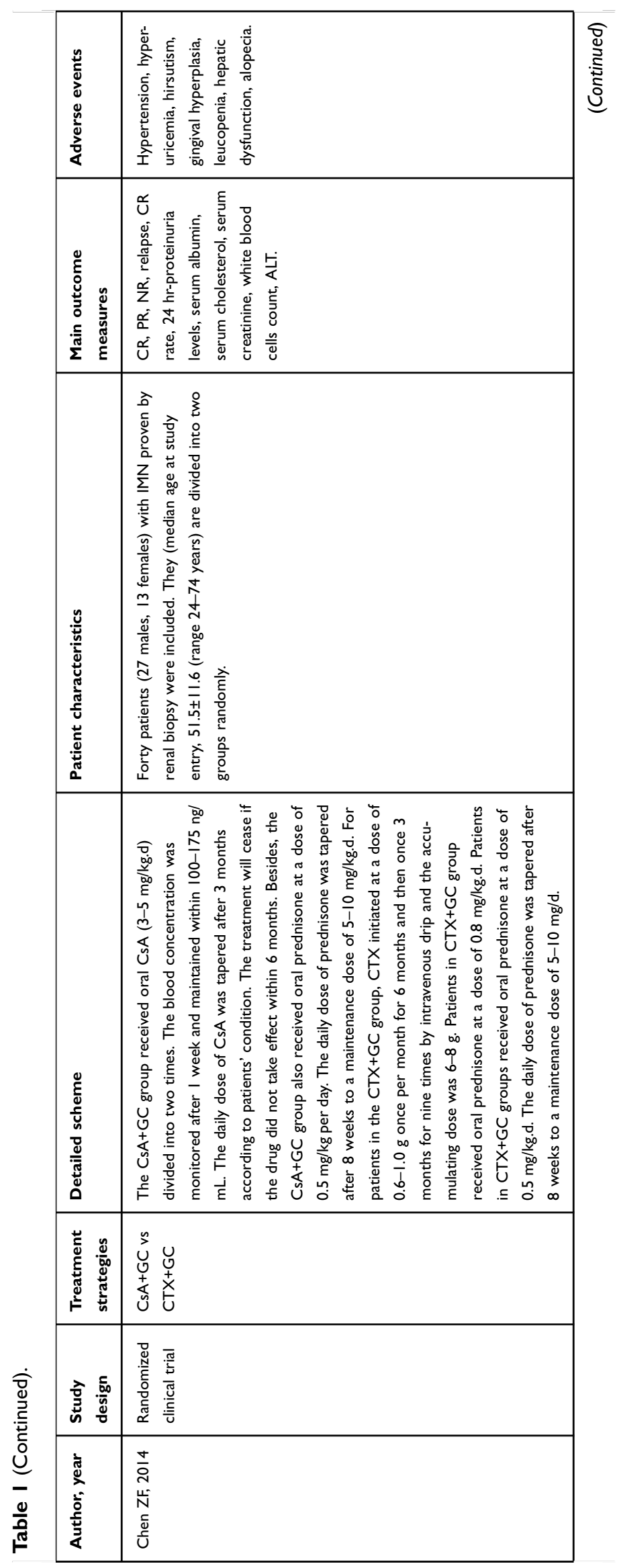




\begin{tabular}{|c|c|c|}
\hline 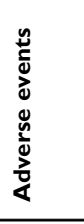 & 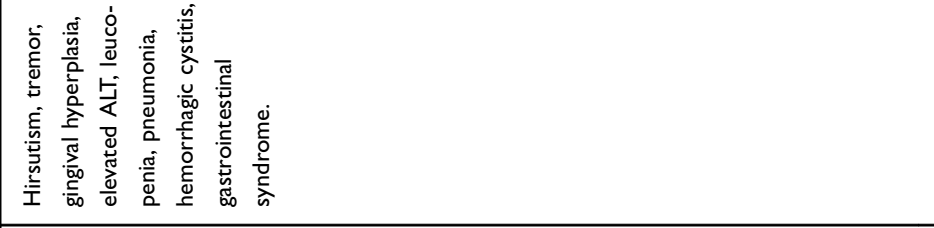 & 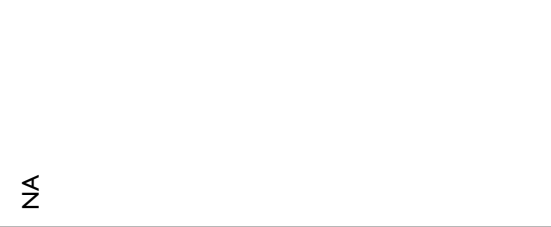 \\
\hline 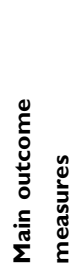 & 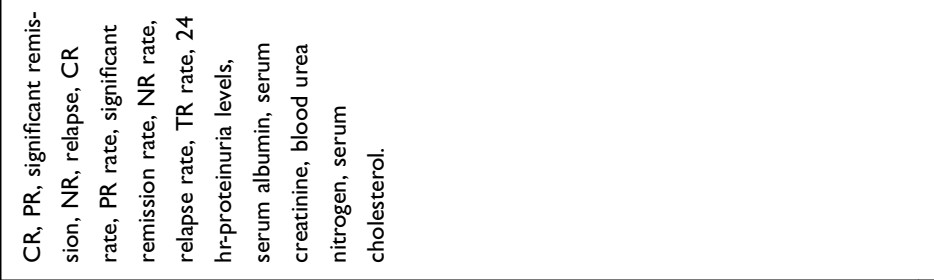 & 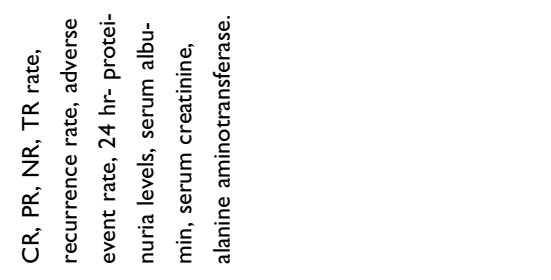 \\
\hline & 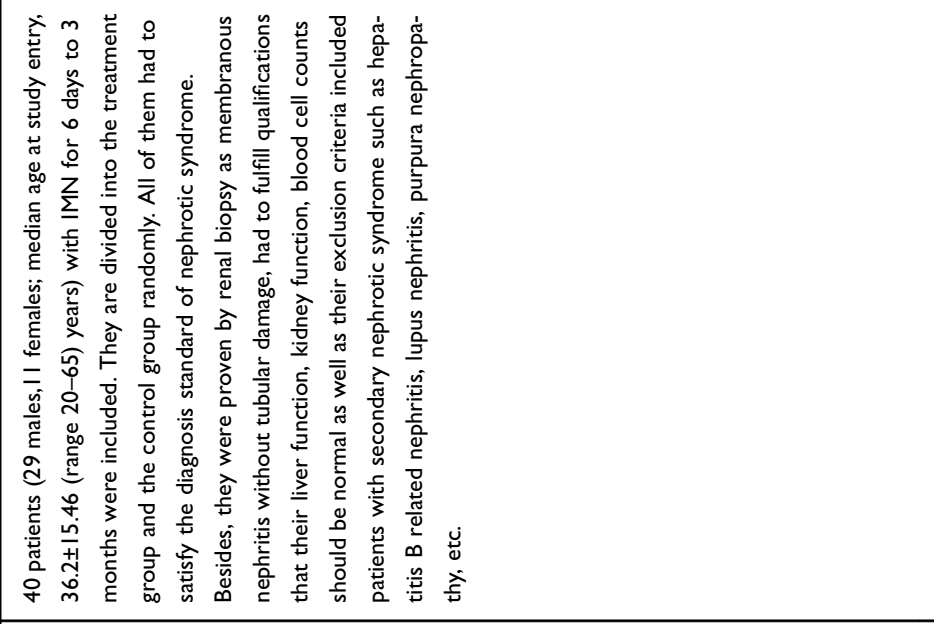 & 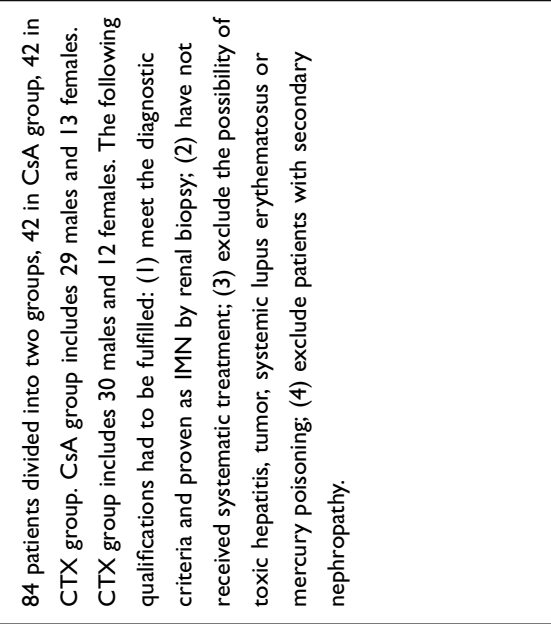 \\
\hline & 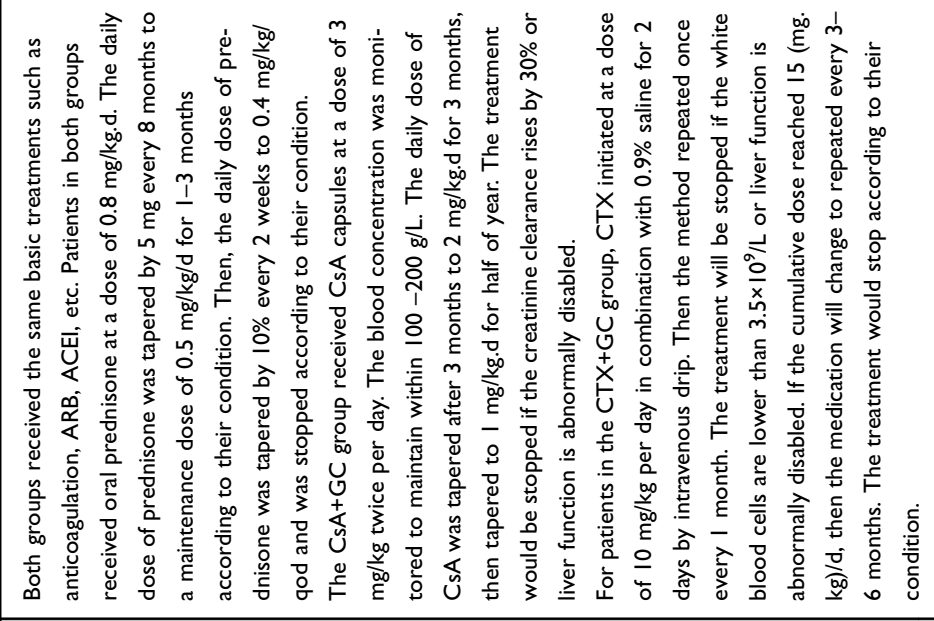 & 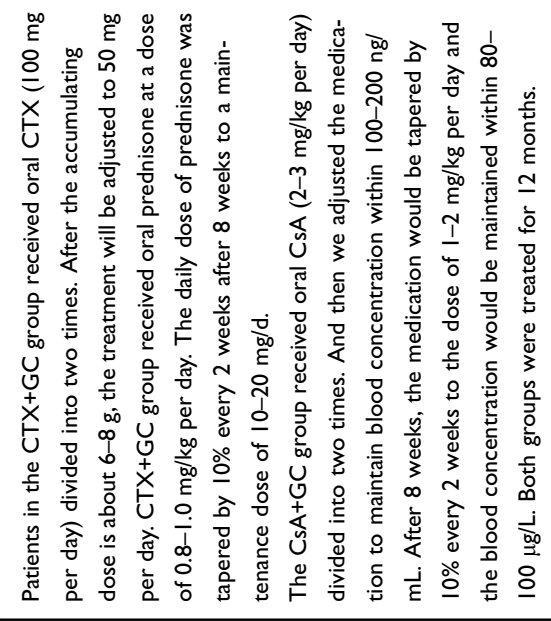 \\
\hline 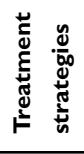 & 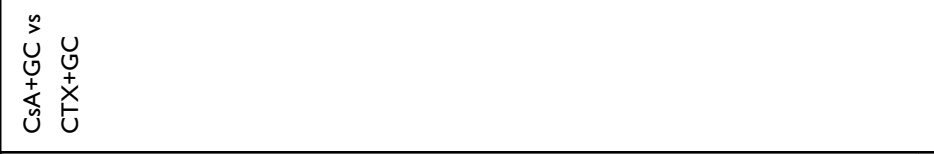 & 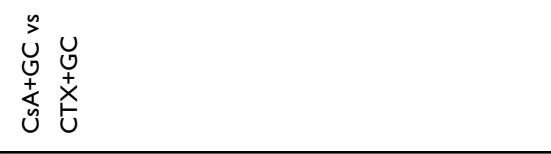 \\
\hline 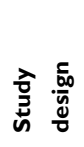 & 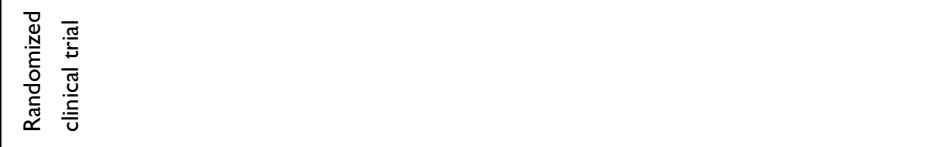 & 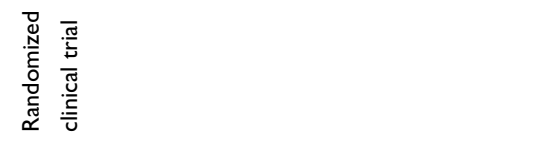 \\
\hline 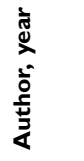 & 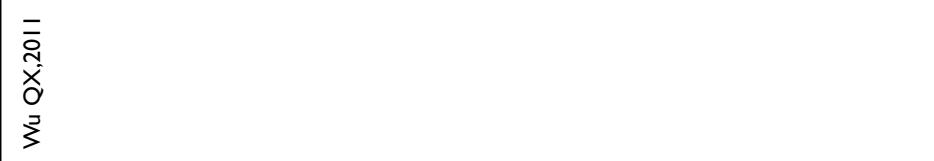 & 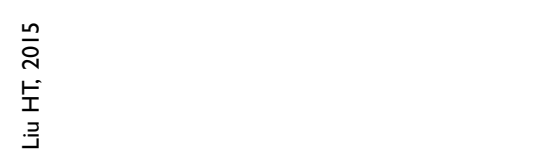 \\
\hline
\end{tabular}




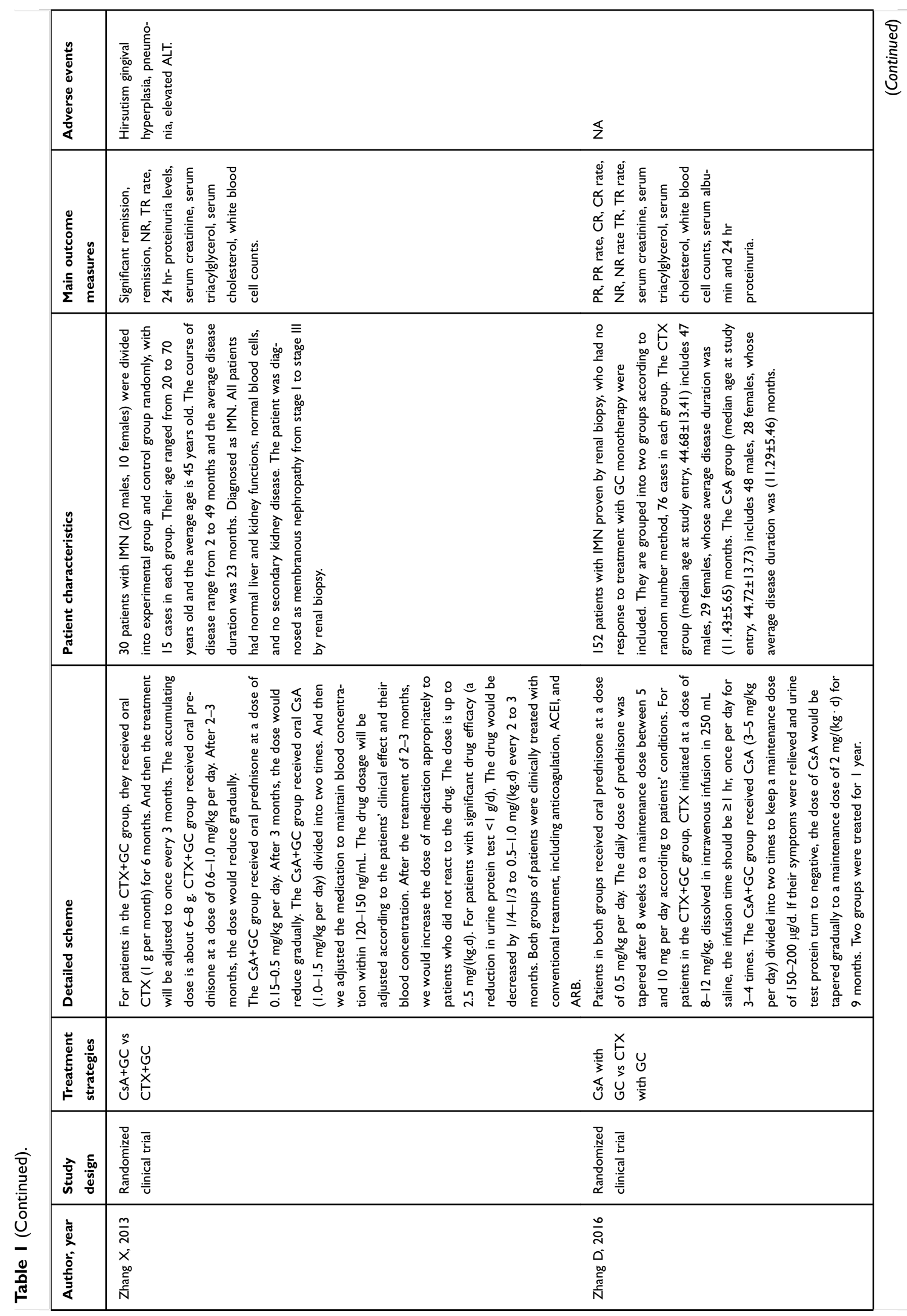




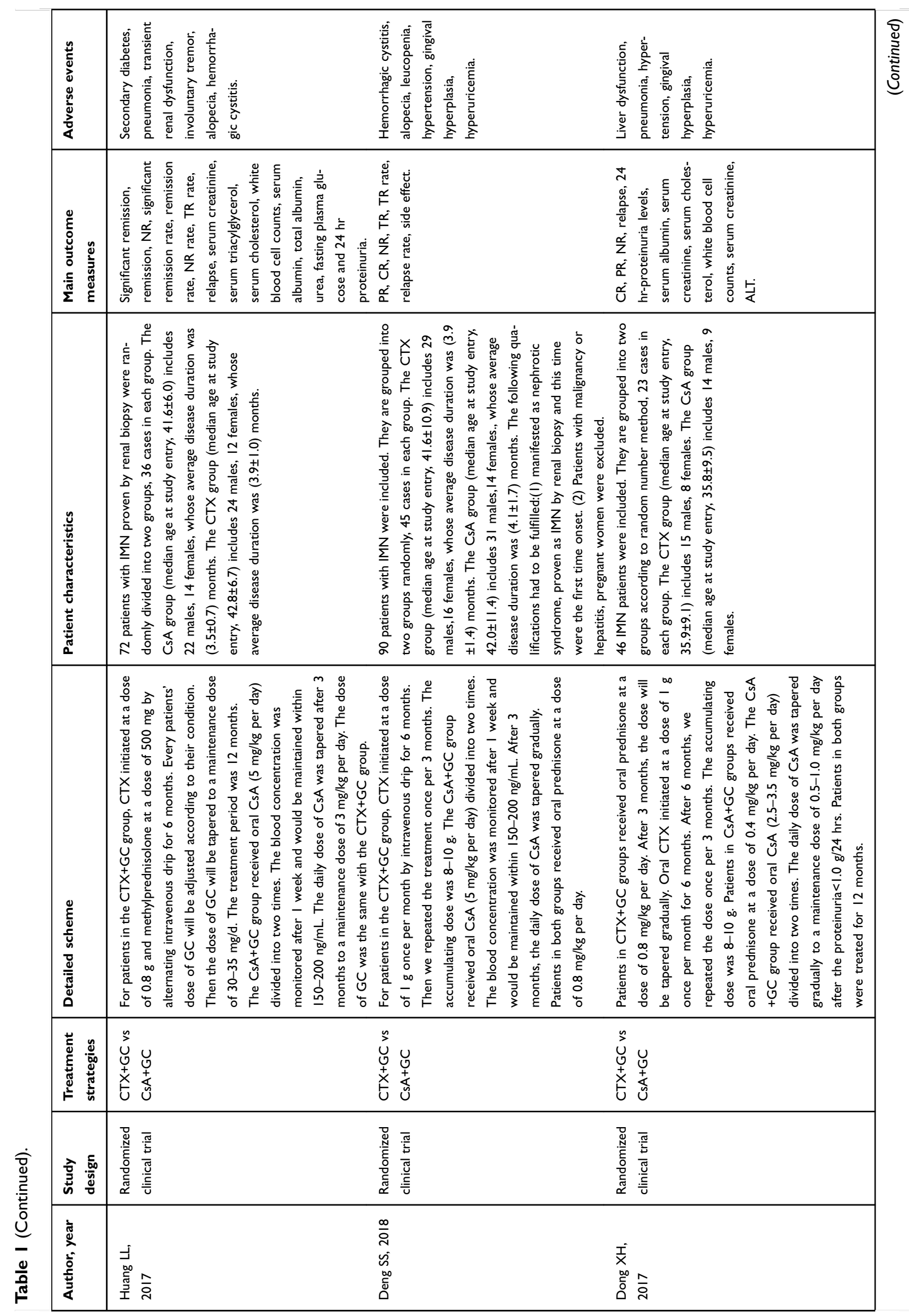




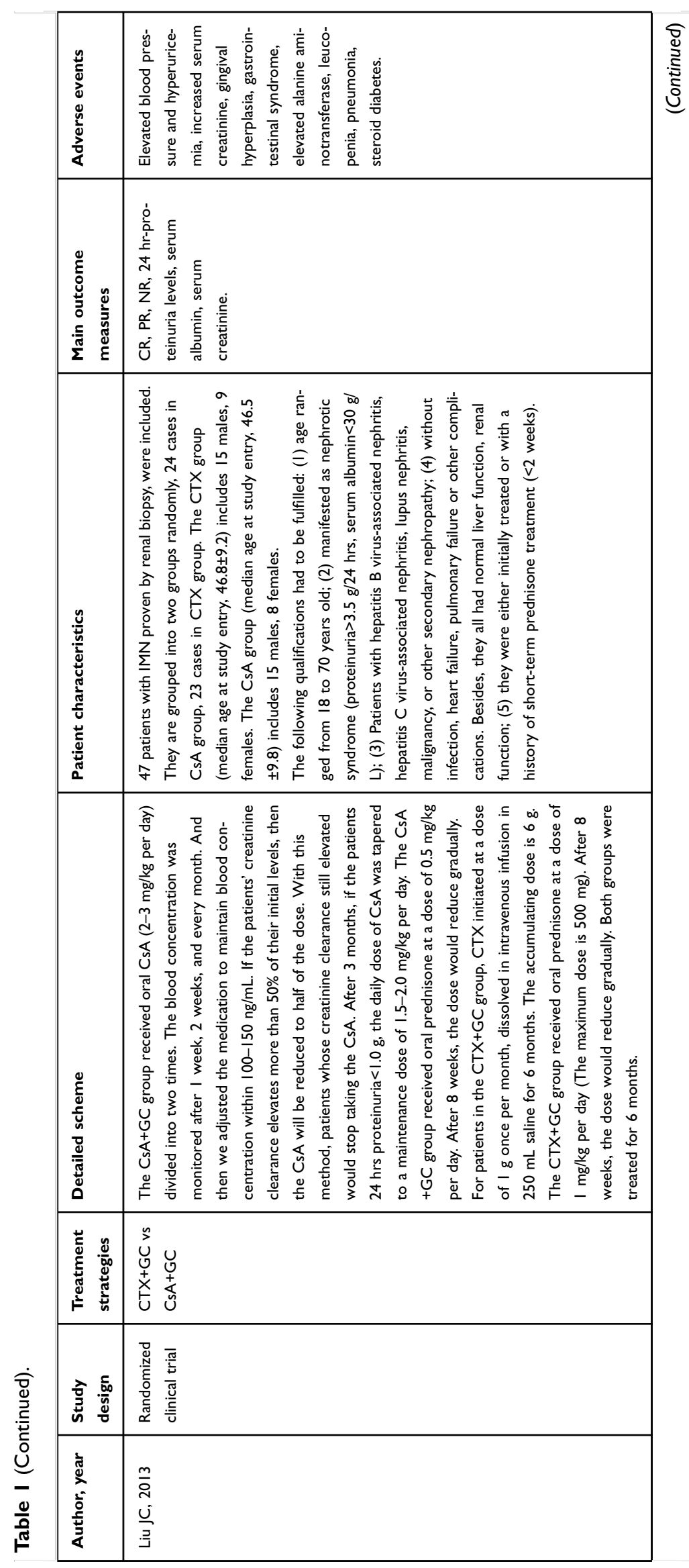




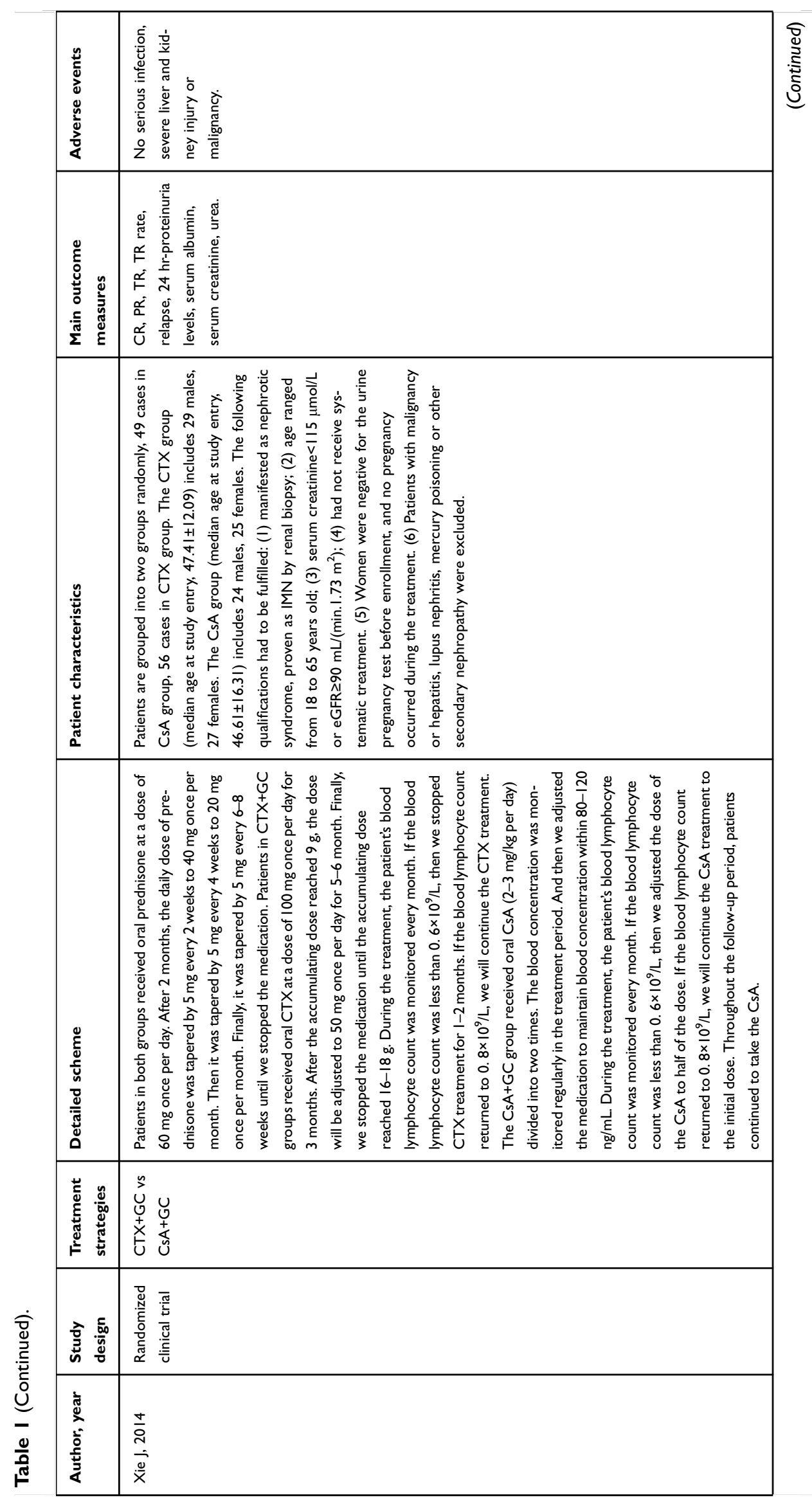




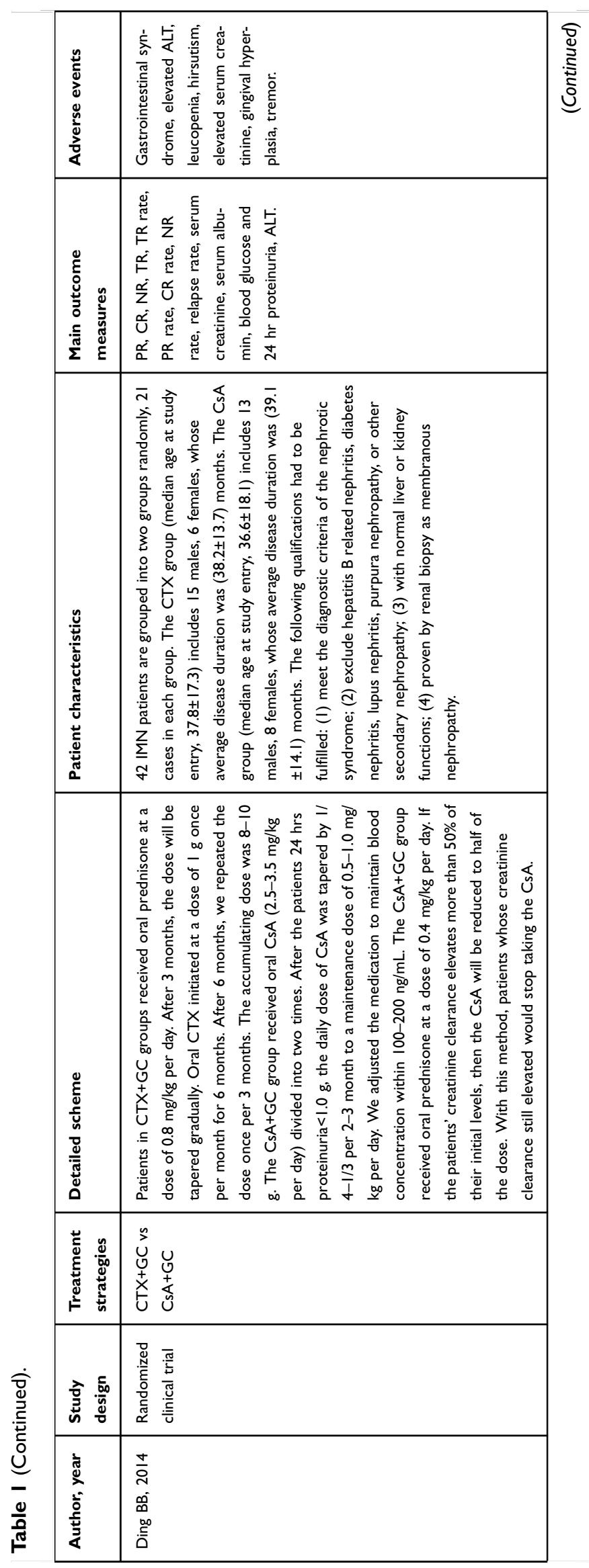




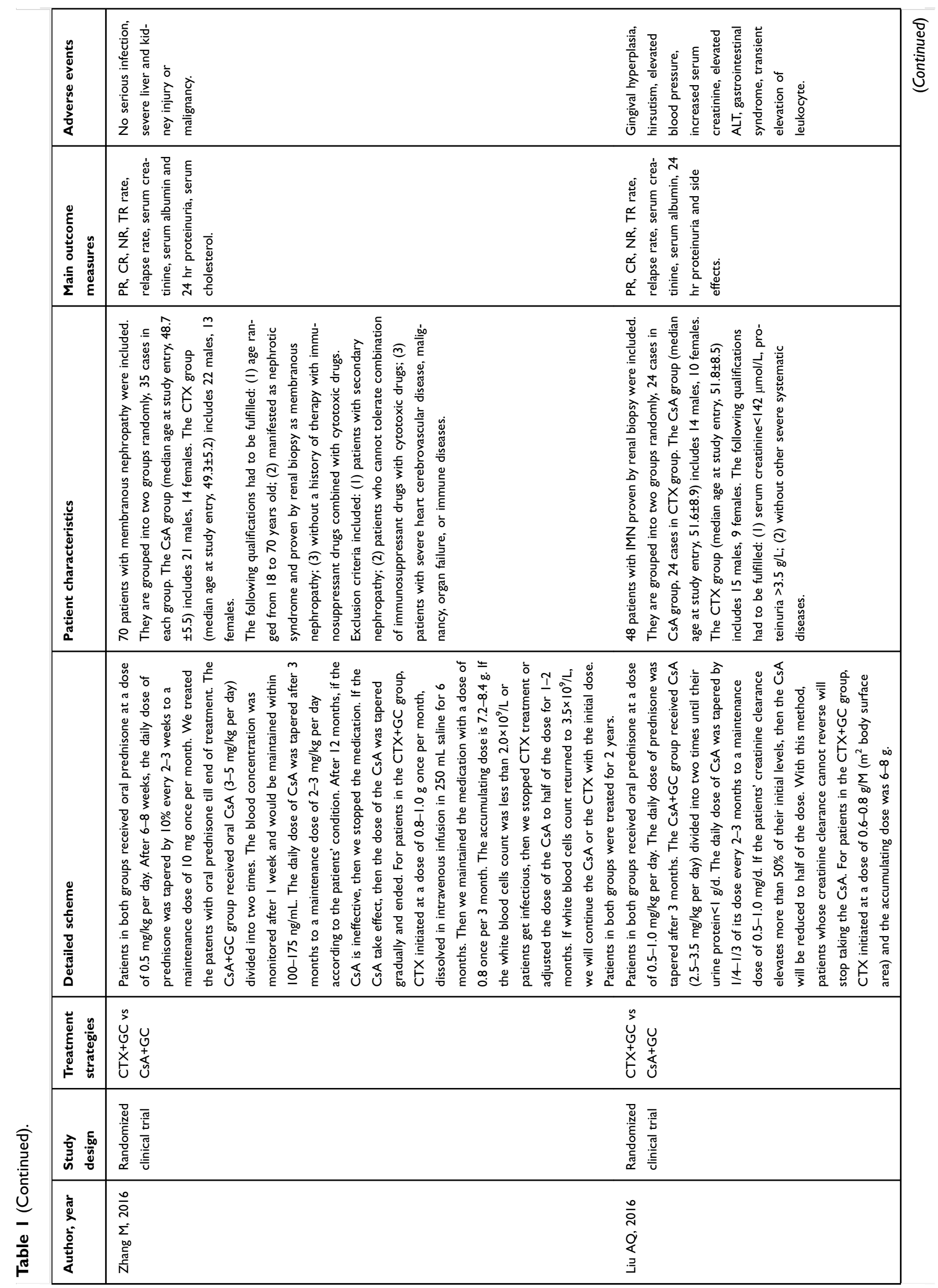




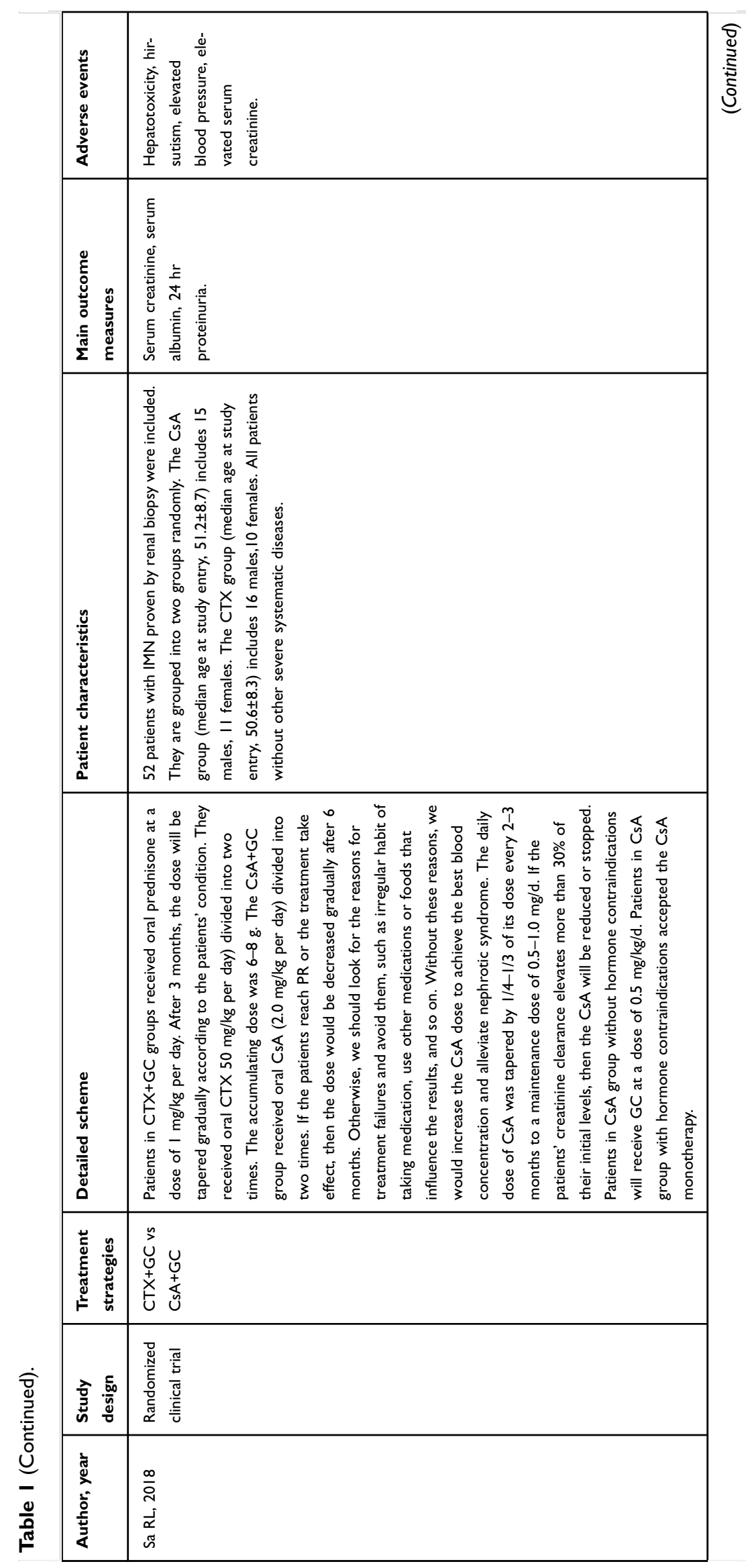




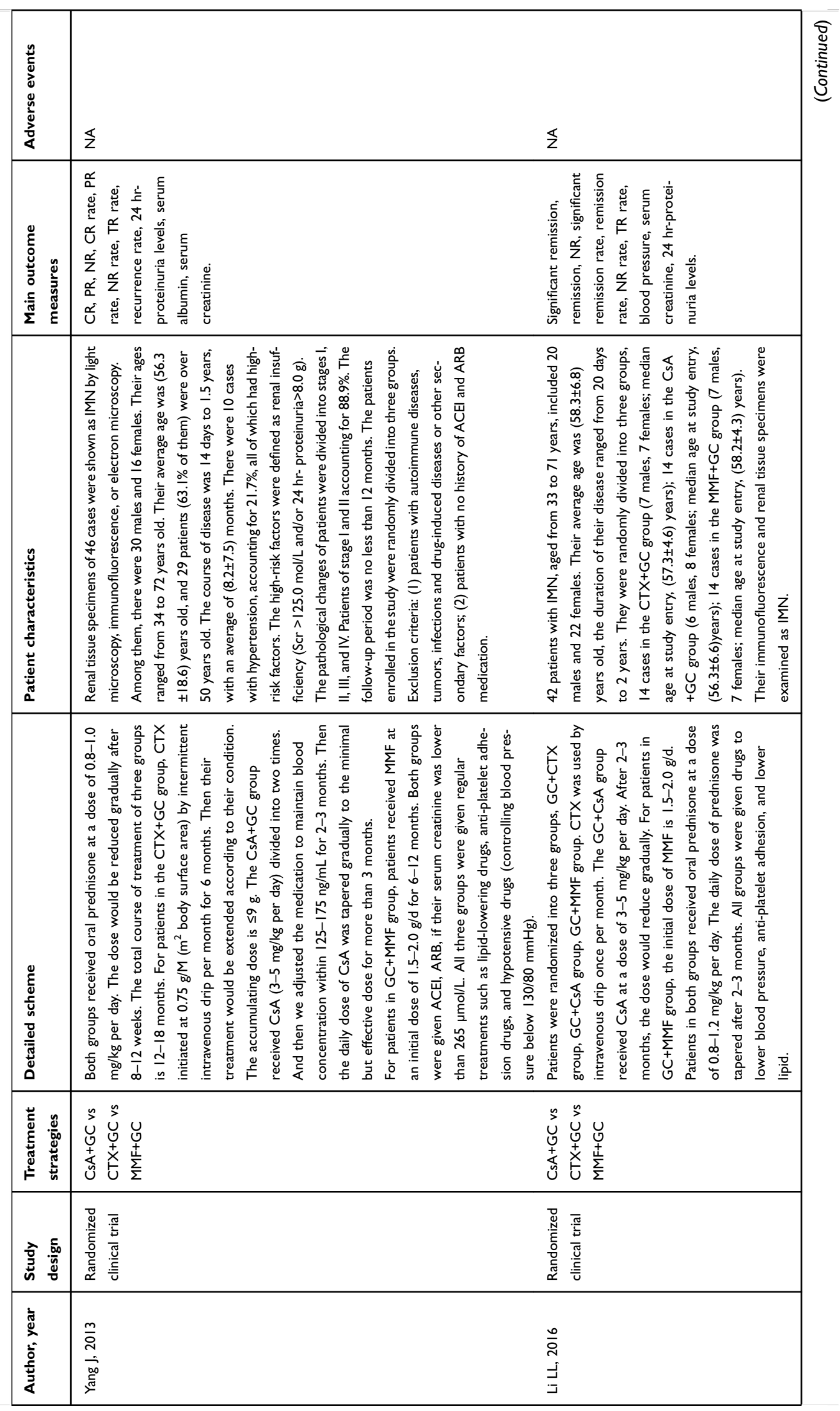



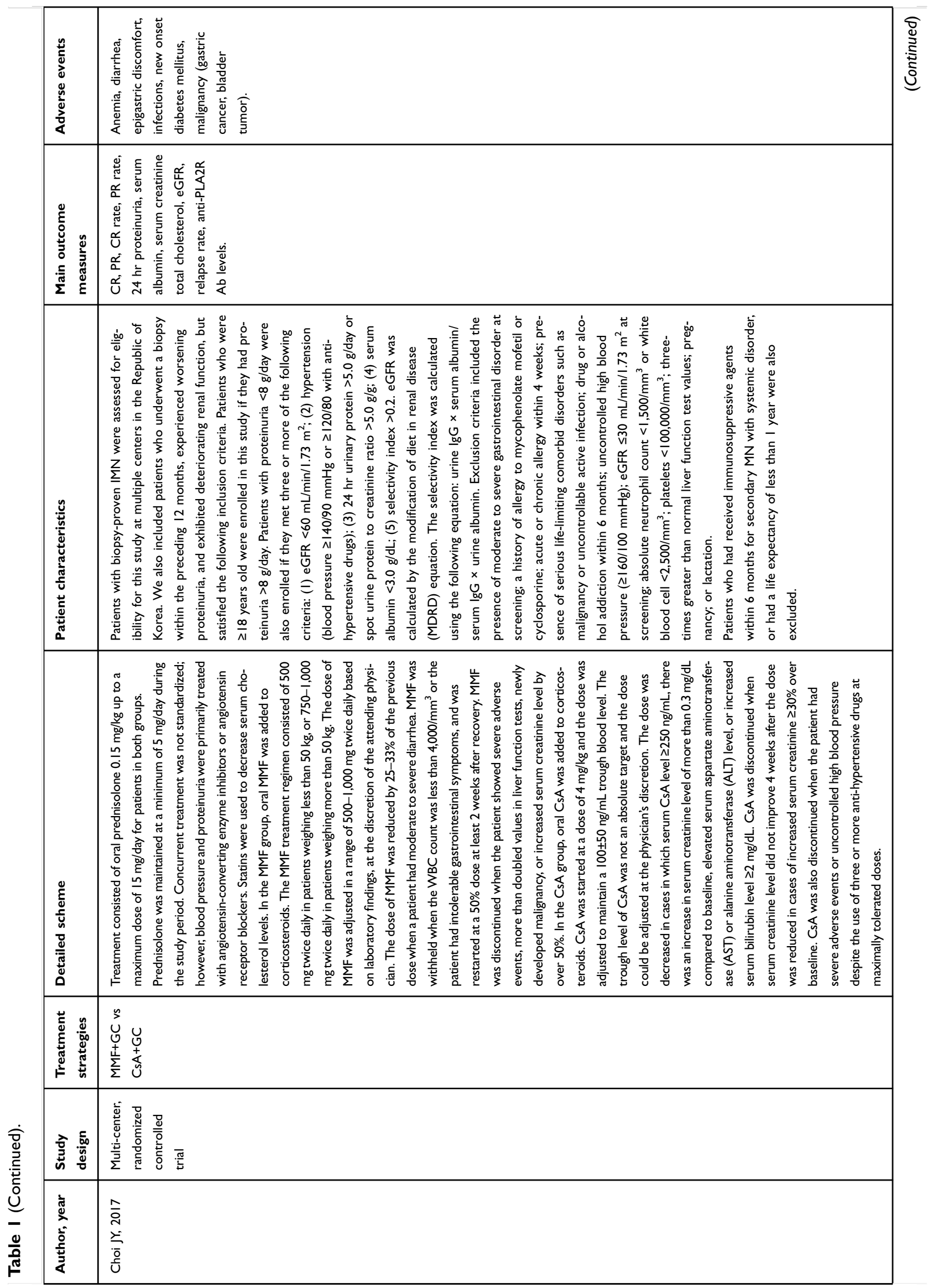


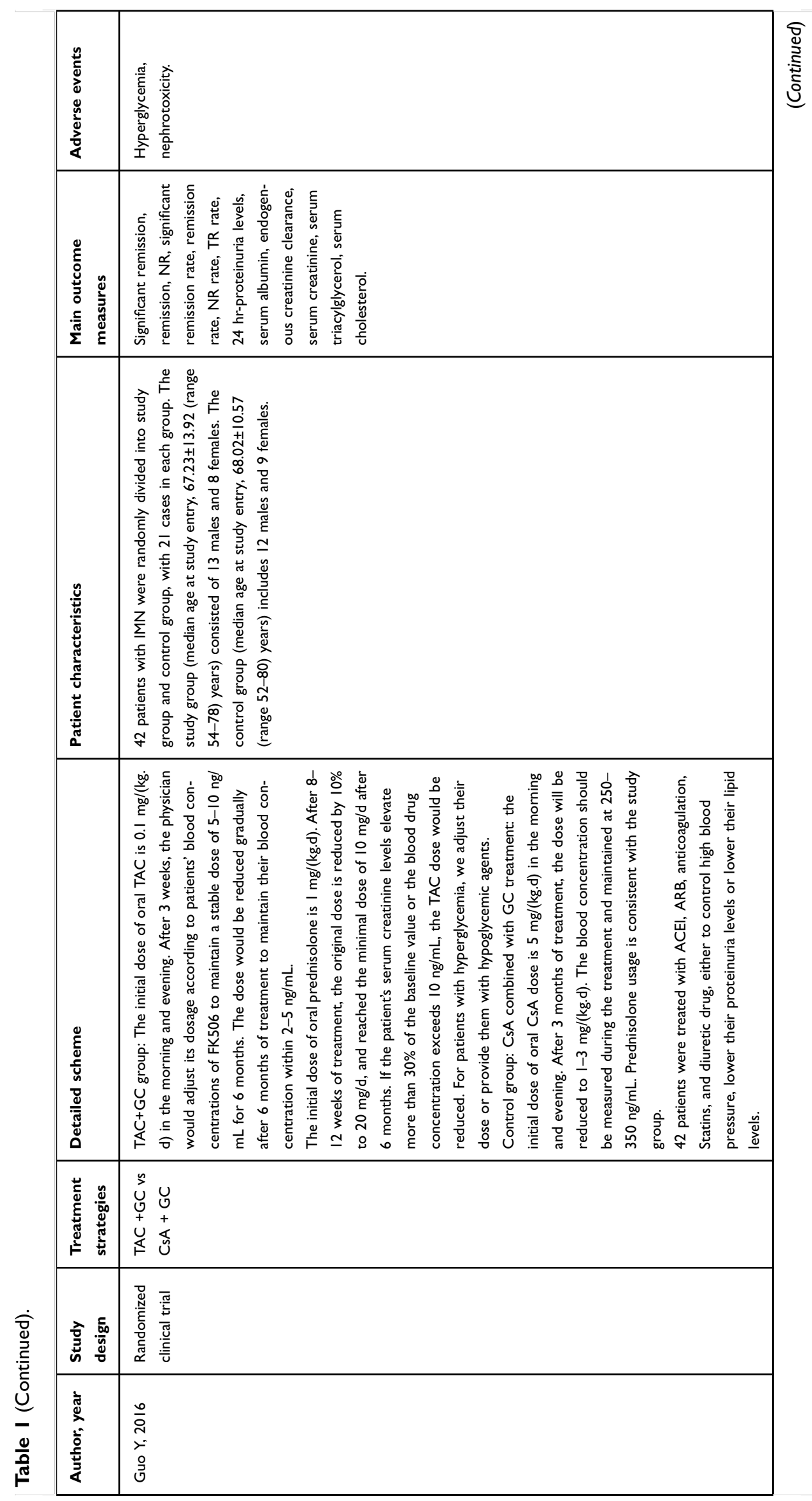




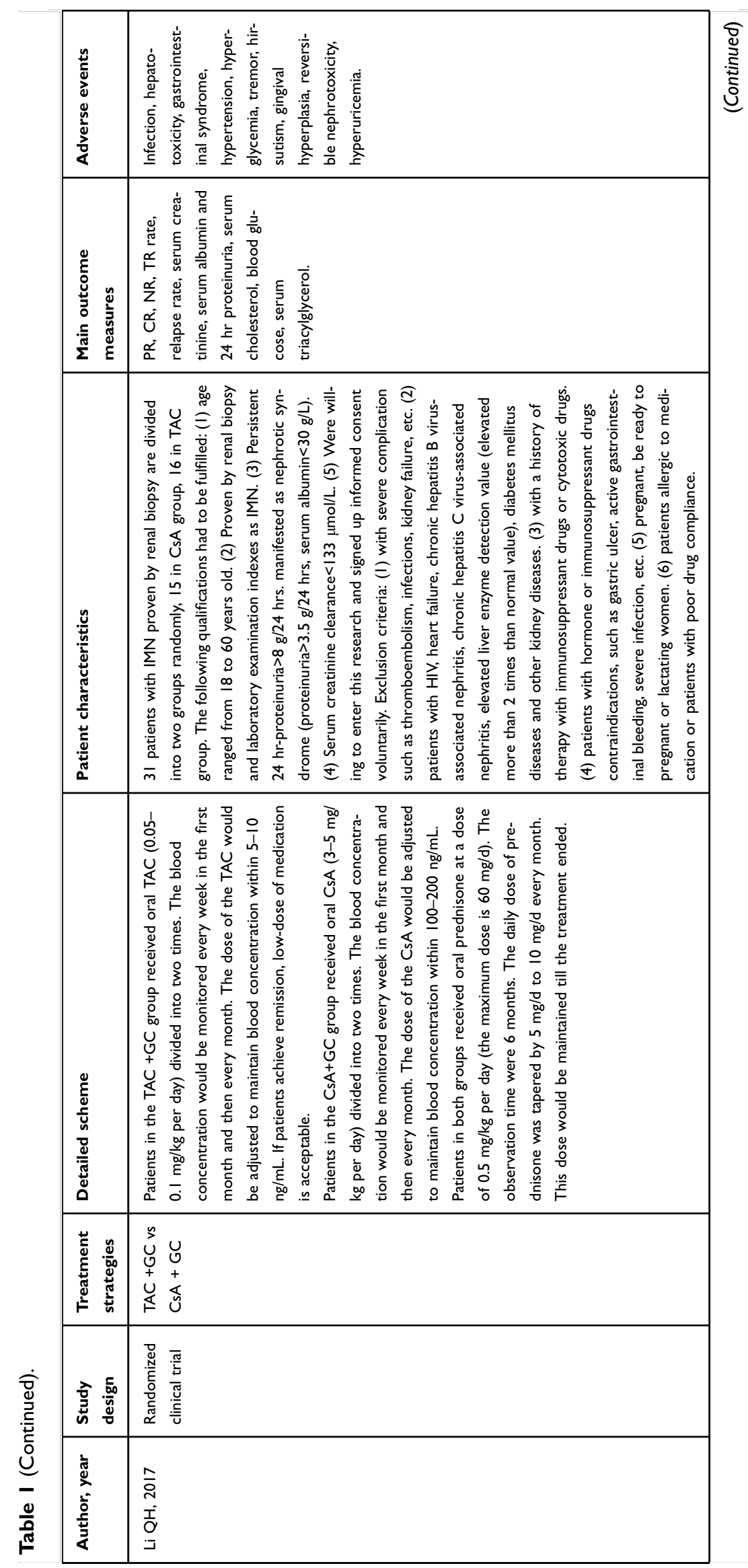




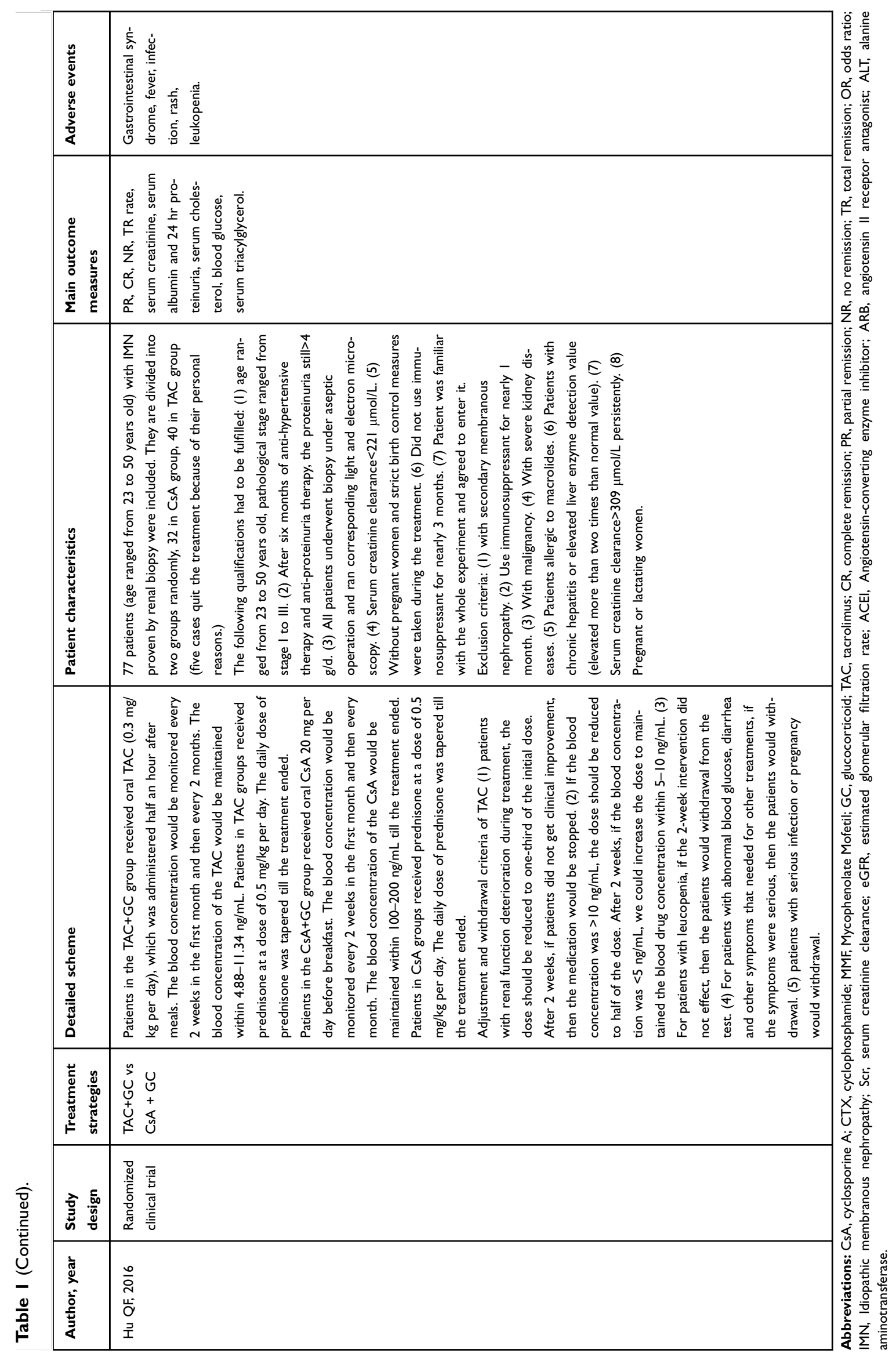




\begin{tabular}{|c|c|c|c|c|c|c|c|c|c|c|}
\hline \multicolumn{11}{|c|}{ CsA+GC vs. CTX+GC on complete remission at 3 months } \\
\hline \multirow[b]{2}{*}{ Study or subgroup } & \multirow{2}{*}{$\begin{array}{r}\text { CsA } \\
\text { Events }\end{array}$} & \multirow{2}{*}{ Total } & \multicolumn{2}{|c|}{ CTX } & \multirow{2}{*}{ Weight } & \multirow{2}{*}{$\begin{array}{c}\text { Odds ratio } \\
\text { M-H, Fixed, } 95 \% \mathrm{Cl}\end{array}$} & \multirow{2}{*}{\multicolumn{4}{|c|}{$\begin{array}{c}\text { Odds ratio } \\
\text { M-H, Fixed, } 95 \% \mathrm{Cl}\end{array}$}} \\
\hline & & & Events & Total & & & & & & \\
\hline Chen ZF 2014 & 6 & 20 & 2 & 20 & $5.5 \%$ & $3.86[0.67,22.11]$ & & & & \\
\hline Ding BB 2014 & 2 & 21 & 3 & 21 & $10.6 \%$ & $0.63[0.09,4.23]$ & & & & \\
\hline Liu AQ 2016 & 6 & 24 & 7 & 24 & $20.5 \%$ & $0.81[0.23,2.90]$ & & & & \\
\hline Liu HT 2015 & 12 & 42 & 3 & 42 & $8.4 \%$ & $5.20[1.35,20.09]$ & & & & \\
\hline Liu JC 2013 & 5 & 22 & 1 & 22 & $3.0 \%$ & $6.18[0.66,58.03]$ & & & & \\
\hline Liu LH 2013 & 2 & 14 & 4 & 16 & $12.5 \%$ & $0.50[0.08,3.27]$ & & & & \\
\hline Xie J 2014 & 11 & 49 & 4 & 56 & $11.3 \%$ & $3.76[1.11,12.73]$ & & & & \\
\hline Zhang M 2013 & 12 & 35 & 8 & 35 & $20.5 \%$ & $1.76[0.61,5.05]$ & & & & \\
\hline Zhang X 2013 & 5 & 15 & 3 & 15 & $7.8 \%$ & $2.00[0.38,10.51]$ & & & & \\
\hline Total $(95 \% \mathrm{Cl})$ & & 242 & & 251 & $100.0 \%$ & $2.07[1.30,3.29]$ & & & & \\
\hline Total events & 61 & & 35 & & & & & & & \\
\hline \multirow{2}{*}{\multicolumn{5}{|c|}{$\begin{array}{l}\text { Heterogeneity: } C h i^{2}=9.98, d f=8(P=0.27) ; I^{2}=20 \% \\
\text { Test for overall effect: } Z=3.08(P=0.002)\end{array}$}} & & & 0.01 & $\begin{array}{ll}0.1 & 1\end{array}$ & 10 & 100 \\
\hline & & & & & & & & Favours $[\mathrm{CsA}]$ & Favours[CTX] & \\
\hline
\end{tabular}

$C s A+G C$ vs. $C T X+G C$ on complete remission at 6 months

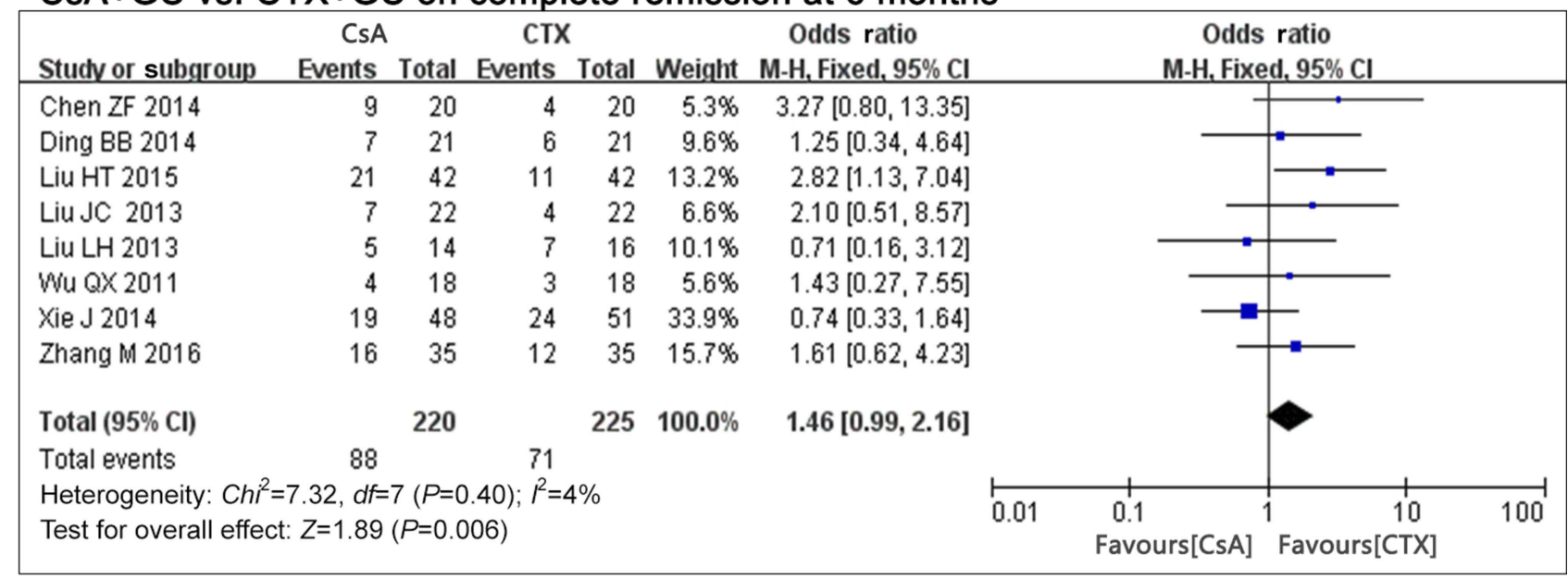

\section{CsA+GC vs. $C T X+G C$ on complete remission at 12 months}

\begin{tabular}{|c|c|c|c|c|c|c|c|c|c|c|}
\hline Study or subgroup & $\begin{array}{r}\text { CsA } \\
\text { Events } \\
\end{array}$ & Total & $\begin{array}{l}\text { CTX } \\
\text { Events }\end{array}$ & Total & Weight & $\begin{array}{c}\text { Odds ratio } \\
\text { M-H, Fixed, } 95 \% \mathrm{Cl}\end{array}$ & & $\begin{array}{r}\text { Odds } \\
\text { M-H, Fixe }\end{array}$ & $\begin{array}{l}\text { ratio } \\
\text { ed, } 95 \% \mathrm{Cl}\end{array}$ & \\
\hline Chen ZF 2014 & 15 & 45 & 17 & 45 & $11.8 \%$ & $0.82[0.35,1.95]$ & & $\longrightarrow$ & & \\
\hline Deng SS 2018 & 11 & 20 & 8 & 20 & $3.7 \%$ & $1.83[0.52,6.43]$ & & & & \\
\hline Ding BB 2014 & 9 & 21 & 10 & 21 & $5.9 \%$ & $0.82[0.24,2.79]$ & & & & \\
\hline Dong XH 2017 & 11 & 23 & 10 & 23 & $5.4 \%$ & $1.19[0.37,3.81]$ & & & & \\
\hline Huang LL 2017 & 10 & 36 & 7 & 36 & $5.3 \%$ & $1.59[0.53,4.79]$ & & & & \\
\hline Huang ZM 2016 & 18 & 50 & 19 & 50 & $12.6 \%$ & $0.92[0.41,2.07]$ & & & & \\
\hline Li LL 2016 & 10 & 14 & 7 & 14 & $2.1 \%$ & $2.50[0.52,11.93]$ & & & & \\
\hline Liu HT 2015 & 25 & 42 & 22 & 42 & $9.2 \%$ & $1.34[0.56,3.17]$ & & & & \\
\hline Liu LH 2013 & 7 & 14 & 8 & 16 & $3.9 \%$ & $1.00[0.24,4.20]$ & & & & \\
\hline Xie J 2014 & 29 & 46 & 41 & 51 & $14.9 \%$ & $0.42[0.17,1.04]$ & & & & \\
\hline Yang J 2013 & 5 & 15 & 4 & 15 & $2.8 \%$ & $1.38[0.29,6.60]$ & & & & \\
\hline Zhang D 2016 & 30 & 76 & 20 & 76 & $12.6 \%$ & $1.83[0.92,3.63]$ & & & & \\
\hline Zhang M 2016 & 18 & 35 & 15 & 35 & $7.6 \%$ & $1.41[0.55,3.62]$ & & & & \\
\hline Zhang $\times 2013$ & 7 & 15 & 4 & 15 & $2.2 \%$ & $2.41[0.52,11.10]$ & & & & \\
\hline Total $(95 \% \mathrm{Cl})$ & & 452 & & 459 & $100.0 \%$ & $1.18[0.90,1.55]$ & & & & \\
\hline \multirow{2}{*}{\multicolumn{7}{|c|}{$\begin{array}{l}\text { Heterogeneity: } C h i^{2}=10.69, d f=13(P=0.64) ; I^{2}=0 \% \\
\text { Test for overall effect: } Z=1.22(P=0.22)\end{array}$}} & & & & \\
\hline & & & & & & & 0.01 & $\begin{array}{l}0.1 \\
\text { Favours }[\mathrm{CsA}]\end{array}$ & $1 \frac{10}{\text { Favours }[\text { CTX] }}$ & 100 \\
\hline
\end{tabular}

Figure I The effect of $C s A+G C$ vs $C T X+G C$ on $C R$ in patients with IMN in Asian populations.

Abbreviations: CsA, cyclosporine A; CTX, cyclophosphamide; GC, glucocorticoid; CR, complete remission; IMN, idiopathic membranous nephropathy. 
Table 2 Meta-analysis of the efficacy of CsA in the therapy of IMN in the Asian population

\begin{tabular}{|c|c|c|c|c|c|c|}
\hline Therapeutic & \multirow[t]{2}{*}{ Indicators } & Studies & $\mathbf{Q}$ test & Model & OR/WMD & \multirow[t]{2}{*}{$\boldsymbol{P}$} \\
\hline Regimen & & Number & $P$-value & Selected & $(95 \% \mathrm{Cl})$ & \\
\hline \multirow[t]{28}{*}{$\mathrm{Cs} A+G C$ vs $\mathrm{CTX}+\mathrm{GC}$} & CR 3 months & 9 & 0.27 & Fixed & $2.07(1.30,3.29)$ & 0.002 \\
\hline & CR 6 months & 8 & 0.40 & Fixed & $1.46(0.99,2.16)$ & 0.06 \\
\hline & CR 12 months & 14 & 0.64 & Fixed & I.18 $(0.90,1.55)$ & 0.22 \\
\hline & NR 3 months & 9 & 0.23 & Fixed & $0.52(0.36,0.74)$ & 0.0004 \\
\hline & NR 6 months & 8 & 0.15 & Fixed & $0.62(0.41,0.93)$ & 0.02 \\
\hline & NR 12 months & 14 & 0.50 & Fixed & $0.5 \mathrm{I}(0.36,0.73)$ & 0.0002 \\
\hline & TR 3 months & 9 & 0.23 & Fixed & $1.94(1.35,2.80)$ & 0.0004 \\
\hline & TR 6 months & 8 & 0.15 & Fixed & $1.62(1.07,2.46)$ & 0.02 \\
\hline & TR 12 months & 14 & 0.39 & Fixed & $1.81(1.29,2.53)$ & 0.0006 \\
\hline & Relapse & 10 & 0.10 & Fixed & $3.06(1.84,5.07)$ & $P<0.0001$ \\
\hline & Serum protein 3 months & 6 & 0.008 & Random & $0.16(-0.27,0.59)$ & 0.48 \\
\hline & Serum protein 6 months & 6 & 0.09 & Random & $0.56(0.21,0.90)$ & 0.002 \\
\hline & Serum protein 12 months & 8 & $<0.00001$ & Random & $1.40(0.15,2.64)$ & 0.03 \\
\hline & Urinary protein 3 months & 6 & 0.007 & Random & $-0.35(-0.78,0.08)$ & 0.11 \\
\hline & Urinary protein 6 months & 6 & 0.02 & Random & $-0.01(-0.4 I, 0.39)$ & 0.96 \\
\hline & Urinary protein 12 months & 8 & $P<0.00001$ & Random & $-0.66(-1.24,-0.08)$ & 0.02 \\
\hline & Serum creatinine 3 months & 6 & 0.29 & Fixed & $0.07(-0.16,0.30)$ & 0.56 \\
\hline & Serum creatinine 6 months & 6 & 0.21 & Fixed & $0.12(-0.12,0.35)$ & 0.34 \\
\hline & Serum creatinine 12 months & 8 & 0.69 & Fixed & $0.13(-0.04,0.31)$ & 0.14 \\
\hline & Cholesterol 3 months & 2 & 0.78 & Fixed & $-0.49(-0.96,-0.01)$ & 0.04 \\
\hline & Cholesterol 6 months & 3 & 0.26 & Fixed & $-0.55(-0.94,-0.16)$ & 0.006 \\
\hline & Cholesterol 12 months & 6 & $<0.00001$ & Random & $-0.55(-1.14,0.03)$ & 0.06 \\
\hline & Triacylglycerol 12 months & 4 & 0.01 & Random & $-0.96(-1.50,-0.4 I)$ & 0.0006 \\
\hline & WBC 12 months & 4 & 0.51 & Fixed & $1.31(1.04,1.58)$ & $<0.00001$ \\
\hline & ALT 3 months & 3 & 0.84 & Fixed & $0.02(-0.28,0.33)$ & 0.88 \\
\hline & ALT 6 months & 3 & 0.99 & Fixed & $0.05(-0.25,0.36)$ & 0.73 \\
\hline & ALT 12 months & 4 & 0.99 & Fixed & $-0.00(-0.27,0.27)$ & 1.00 \\
\hline & BUN & 2 & 0.82 & Fixed & $-0.00(-0.38,0.38)$ & 0.99 \\
\hline \multirow[t]{3}{*}{ CsA vs MMF } & $C R$ & 3 & 0.21 & Fixed & $0.92(0.41,2.05)$ & 0.83 \\
\hline & NR & 3 & 0.13 & Fixed & $0.55(0.22,1.36)$ & 0.20 \\
\hline & TR & 3 & 0.13 & Fixed & I.82 $(0.73,4.49)$ & 0.20 \\
\hline \multirow[t]{7}{*}{ CsA vs TAC } & $C R$ & 3 & 0.36 & Fixed & $0.42(0.21,0.85)$ & 0.02 \\
\hline & $N R$ & 3 & 0.96 & Fixed & $3.27(1.40,7.64)$ & 0.006 \\
\hline & $T R$ & 3 & 0.96 & Fixed & $0.31(0.13,0.71)$ & 0.006 \\
\hline & Serum protein & 2 & 0.01 & Random & $-1.17(-2.23,-0.12)$ & 0.03 \\
\hline & Urinary protein & 2 & 0.32 & Fixed & $0.36(-0.01,0.73)$ & 0.06 \\
\hline & Triacylglycerol & 2 & 0.02 & Random & $1.01(0.11,1.92)$ & 0.03 \\
\hline & Cholesterol & 2 & 0.14 & Fixed & $0.34(-0.03,0.72)$ & 0.07 \\
\hline
\end{tabular}

Abbreviations: CsA, cyclosporine A; CTX, cyclophosphamide; MMF, Mycophenolate Mofetil; GC, glucocorticoid; TAC, tacrolimus; CR, complete remission; PR, partial remission; NR, no remission; TR, total remission, $C R$ or $P R ; O R$, odds ratio.

Two studies ${ }^{36,37}$ compared CsA $+\mathrm{GC}$ vs TAC $+\mathrm{GC}$. The CsA had lower levels of serum albumin $(P=0.03, \mathrm{MD}=$ $-1.17,95 \%$ CI: $-2.23,-0.12$; Table 2).

\section{Serum creatinine}

In comparing $\mathrm{CsA}+\mathrm{GC}$ vs $\mathrm{CTX}+\mathrm{GC}$, serum creatinine levels at 3 months ${ }^{17,19,25-28}(P=0.56, \mathrm{MD}=0.07,95 \% \mathrm{CI}:-0.16,0.30$; Table 2), 6 months $^{17,19,26-28,30}(P=0.34, \mathrm{MD}=0.12,95 \% \mathrm{CI}$ :
$-0.12,0.35$; Table 2) and 12 months ${ }^{17,19-21,27,28,32,34}$ $(P=0.14, \mathrm{MD}=0.13,95 \% \mathrm{CI}:-0.04$ to 0.31 ; Table 2$)$ indicated that the CsA group had similar efficacy to the CTX group in preserving renal function.

\section{Serum cholesterol}

In comparing $\mathrm{CsA}+\mathrm{GC}$ vs $\mathrm{CTX}+\mathrm{GC}$, serum cholesterol of the CsA group at 3 months ${ }^{17,27}(P=0.04, \mathrm{MD}=-0.49,95 \%$ 


\begin{tabular}{|c|c|c|c|c|c|c|c|c|c|c|}
\hline \multicolumn{11}{|c|}{ CsA+GC vs. CTX+GC on total remission at 3 months } \\
\hline \multirow[b]{2}{*}{ Study or subgroup } & \multirow{2}{*}{$\begin{array}{l}\text { CsA } \\
\text { Events }\end{array}$} & \multirow{2}{*}{\multicolumn{2}{|c|}{$\begin{array}{r}\text { CTX } \\
\text { Total Events }\end{array}$}} & & \multirow{2}{*}{$\begin{array}{l}\text { Odds ratio } \\
\text { M-H, Fixed, } 95 \% \mathrm{Cl}\end{array}$} & \multirow{2}{*}{\multicolumn{4}{|c|}{$\begin{array}{l}\text { Odds ratio } \\
\text { M-H, Fixed, } 95 \% \mathrm{Cl}\end{array}$}} \\
\hline & & & & Total & Weight & & & & & \\
\hline Chen ZF 2014 & 14 & 20 & 6 & 20 & $4.4 \%$ & $5.44[1.41,21.05]$ & & & & \\
\hline Ding BB 2014 & 10 & 21 & 12 & 21 & $15.2 \%$ & $0.68[0.20,2.30]$ & & & & \\
\hline Liu AQ 2016 & 16 & 24 & 17 & 24 & $13.7 \%$ & $0.82[0.24,2.80]$ & & & & \\
\hline Liu HT 2015 & 19 & 42 & 11 & 42 & $14.6 \%$ & $2.33[0.93,5.83]$ & & & & \\
\hline Liu JC 2013 & 11 & 22 & 4 & 22 & $4.8 \%$ & $4.50[1.15,17.68]$ & & & & \\
\hline Liu LH 2013 & 8 & 14 & 10 & 16 & $9.7 \%$ & $0.80[0.18,3.46]$ & & & & \\
\hline Xie J 2014 & 23 & 49 & 17 & 56 & $20.4 \%$ & $2.03[0.91,4.51]$ & & & & \\
\hline Zhang M 2016 & 25 & 35 & 18 & 35 & $12.4 \%$ & $2.36[0.88,6.34]$ & & & & \\
\hline Zhang $\times 2013$ & 10 & 15 & 6 & 15 & $4.8 \%$ & $3.00[0.68,13.31]$ & & & & \\
\hline Total $(95 \% \mathrm{Cl})$ & & 242 & & 251 & $100.0 \%$ & $1.94[1.35,2.80]$ & & & & \\
\hline \multirow{2}{*}{\multicolumn{7}{|c|}{$\begin{array}{l}\text { Heterogeneity: } C h i^{2}=10.46, d f=8(P=0.23) ; I^{2}=24 \% \\
\text { Test for overall effect: } Z=3.55(P=0.0004)\end{array}$}} & & & & \\
\hline & & & & & & & 0.01 & $\begin{array}{l}0.1 \\
\text { Favours }[\mathrm{Cs} \mathrm{A}]\end{array}$ & $\begin{array}{c}10 \\
\text { Favours }[\mathrm{CTX}]\end{array}$ & 100 \\
\hline
\end{tabular}

\section{CsA+GC vs. $C T X+G C$ on total remission at 6 months}

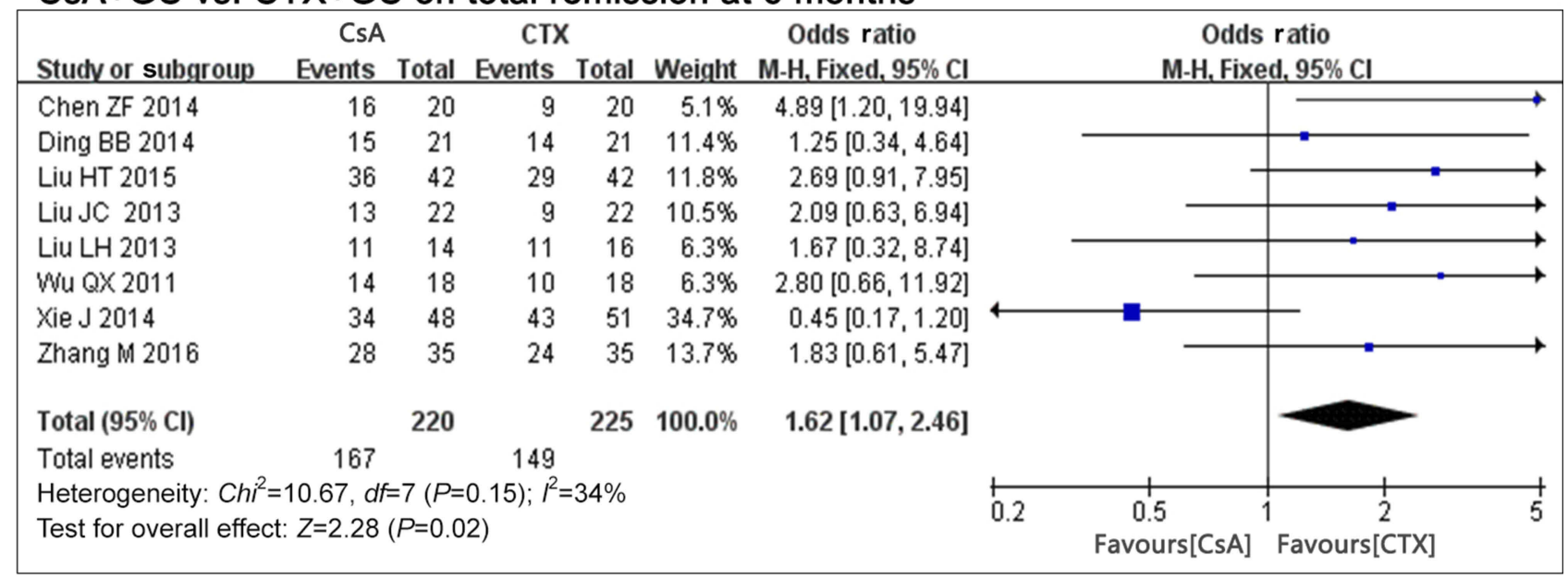

CsA+GC vs. CTX+GC on total remission at 12 months

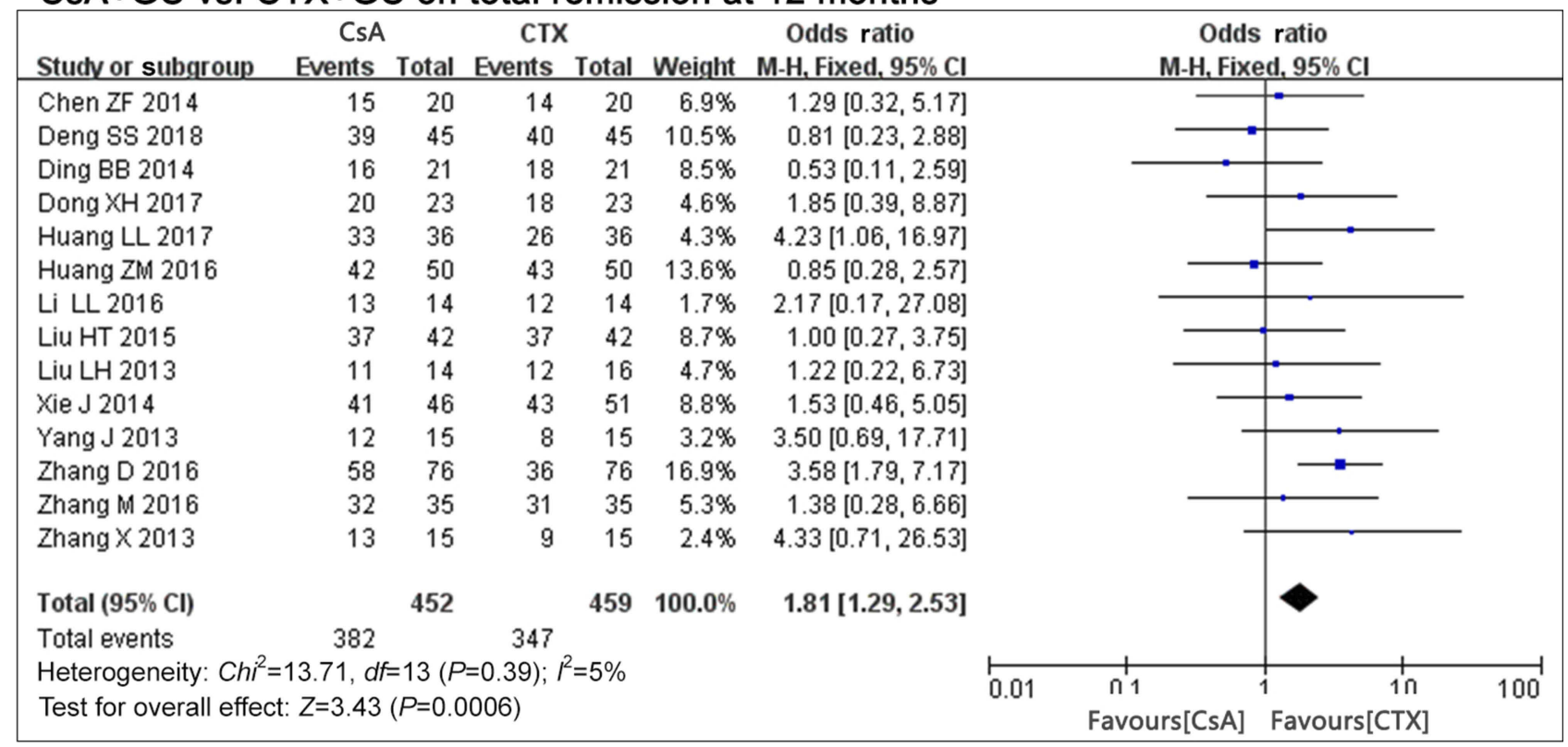

Figure 2 The effect of $C s A+G C$ vs $C T X+G C$ on $T R$ in patients with IMN in Asian populations.

Abbreviations: CsA, cyclosporine A; CTX, cyclophosphamide; GC, glucocorticoid; TR, total remission; IMN, idiopathic membranous nephropathy. 
$C s A+G C$ vs. $C T X+G C$ on proteinuria at 3 months

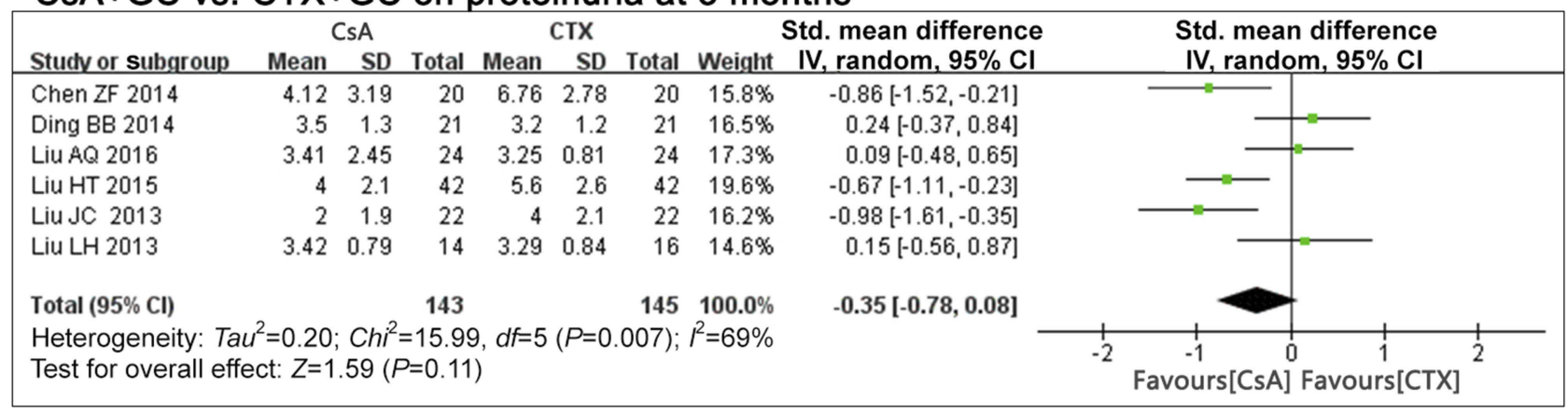

$C s A+G C$ vs. $C T X+G C$ on proteinuria at 6 months

\begin{tabular}{|c|c|c|c|c|c|c|c|c|c|}
\hline \multirow[b]{2}{*}{ Study or subgroup } & \multicolumn{3}{|c|}{ CsA } & \multicolumn{3}{|c|}{ CTX } & \multirow{2}{*}{\multicolumn{2}{|c|}{$\begin{array}{r}\text { Std. mean difference } \\
\text { Weight } \quad \mathrm{IV} \text {, random, } 95 \% \mathrm{CI}\end{array}$}} & \multirow{2}{*}{$\begin{array}{l}\text { Std. mean difference } \\
\mathrm{IV} \text {, random, } 95 \% \mathrm{Cl}\end{array}$} \\
\hline & Mean & SD & Total & Mean & SD & Total & & & \\
\hline Chen ZF 2014 & 3.34 & 3.05 & 20 & 3.91 & 3.15 & 20 & $16.4 \%$ & $-0.18[-0.80,0.44]$ & $x_{1}$ \\
\hline Ding BB 2014 & 1.6 & 0.6 & 21 & 1.2 & 0.5 & 21 & $16.3 \%$ & $0.71[0.09,1.34]$ & \\
\hline Liu HT 2015 & 1.9 & 1.7 & 22 & 2.5 & 2 & 22 & $16.9 \%$ & $-0.32[-0.91,0.28]$ & \\
\hline Liu JC 2013 & 1.35 & 0.64 & 14 & 0.92 & 0.69 & 16 & $14.1 \%$ & $0.63[-0.11,1.36]$ & \\
\hline Liu LH 2013 & 3.2 & 1.6 & 42 & 4 & 2.2 & 42 & $20.7 \%$ & $-0.41[-0.84,0.02]$ & \\
\hline Wu $Q \times 2011$ & 0.33 & 1.5 & 18 & 0.88 & 2.1 & 18 & $15.6 \%$ & $-0.29[-0.95,0.36]$ & \\
\hline \multirow{3}{*}{\multicolumn{8}{|c|}{$\begin{array}{l}\text { Total }(\mathbf{9 5} \% \mathrm{Cl}) \\
\text { Heterogeneity: } \text { Tau }^{2}=0.15 ; C h i^{2}=13.05, d f=5(P=0.02) ; I^{2}=62 \% \\
\text { Test for overall effect: } Z=0.05(P=0.96)\end{array}$}} & $-0.01[-0.41,0.39]$ & \\
\hline & & & & & & & & & $\begin{array}{ccccc}1 & 1 & 1 & 1 & 1 \\
-2 & -1 & 0 & 1 & 2\end{array}$ \\
\hline & & & & & & & & & Favours [CsA] Favours[CTX] \\
\hline
\end{tabular}

CsA+GC vs. CTX+GC on proteinuria at 12 months

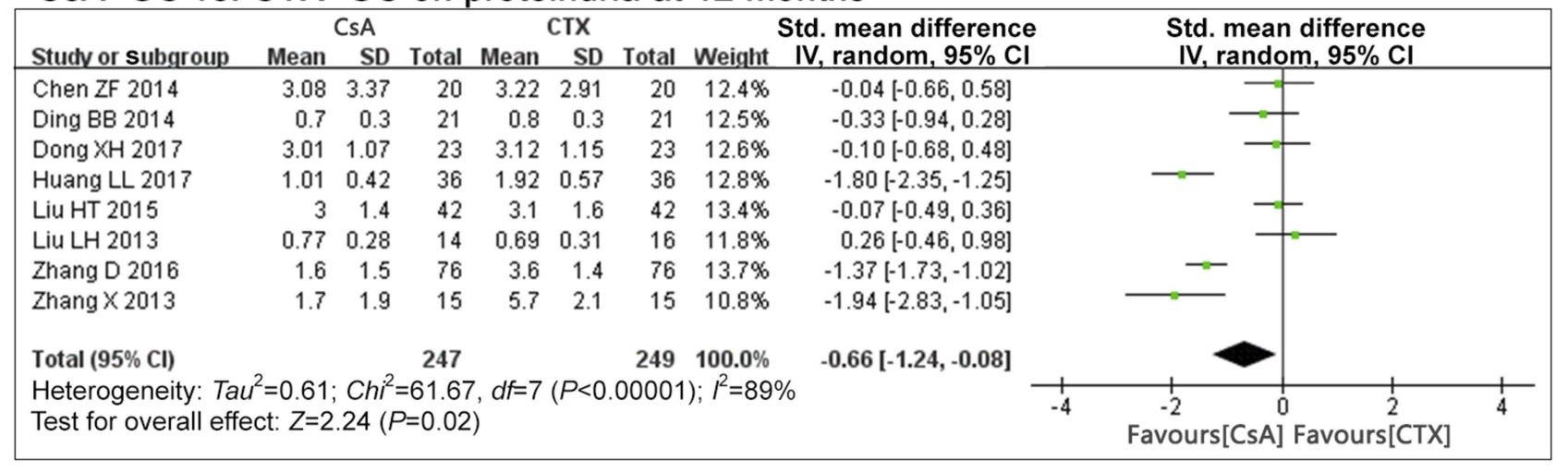

Figure 3 The effect of $C s A+G C$ vs $C T X+G C$ on proteinuria in patients with IMN in Asian populations.

Abbreviations: CsA, cyclosporine A; CTX, cyclophosphamide; GC, glucocorticoid; IMN, idiopathic membranous nephropathy.

CI:-0.96, -0.01; Table 2) and at 6 months ${ }^{17,27,30}(P=0.006$, $\mathrm{MD}=-0.55,95 \% \mathrm{CI}:-0.94,-0.16$; Table 2$)$ was significantly different than the CTX group. This difference was not present at 12 months $^{17,20,21,27,32,34}(P=0.06, \mathrm{MD}=$ $-0.55,95 \% \mathrm{CI}:-1.14$ to 0.03 ; Table 2 ).

Two studies ${ }^{36,37}$ assessed the cholesterol levels of CsA $+\mathrm{GC}$ vs $\mathrm{TAC}+\mathrm{GC}$, which were not different $(P=0.07$, $\mathrm{MD}=0.34,95 \% \mathrm{CI}:-0.03$ to 0.72 ; Table 2 ).

\section{Serum triacylglycerol}

In comparing $\mathrm{CsA}+\mathrm{GC}$ vs $\mathrm{CTX}+\mathrm{GC}$, four studies ${ }^{21,27,32,34}$ assessed serum triacylglycerol at 12 months. CsA was consistent in its effects $(P=0.0006$ at 12 months; Table 2). Its pooled mean difference was as follows: -0.96 (95\% CI:-1.50, -0.41; Table 2) at 12 months.

The comparison of $\mathrm{CsA}+\mathrm{GC}$ vs $\mathrm{TAC}+\mathrm{GC}$ in two studies $^{36,37}$ indicated that the $\mathrm{TAC}+\mathrm{GC}$ group had lower serum triacylglycerol levels $(P=0.03, \mathrm{MD}=1.01,95 \%$ CI:0.11 to 1.92; Table 2).

\section{Other clinical indicators of $\mathrm{Cs} A+G C$ vs CTX+GC}

Comparison of $\mathrm{CsA}+\mathrm{GC}$ vs $\mathrm{CTX}+\mathrm{GC}$, revealed difference in alanine aminotransferase (ALT) at 3 months $(P=0.88$, 
CsA+GC vs. $C T X+G C$ on serum albumin at 3 months

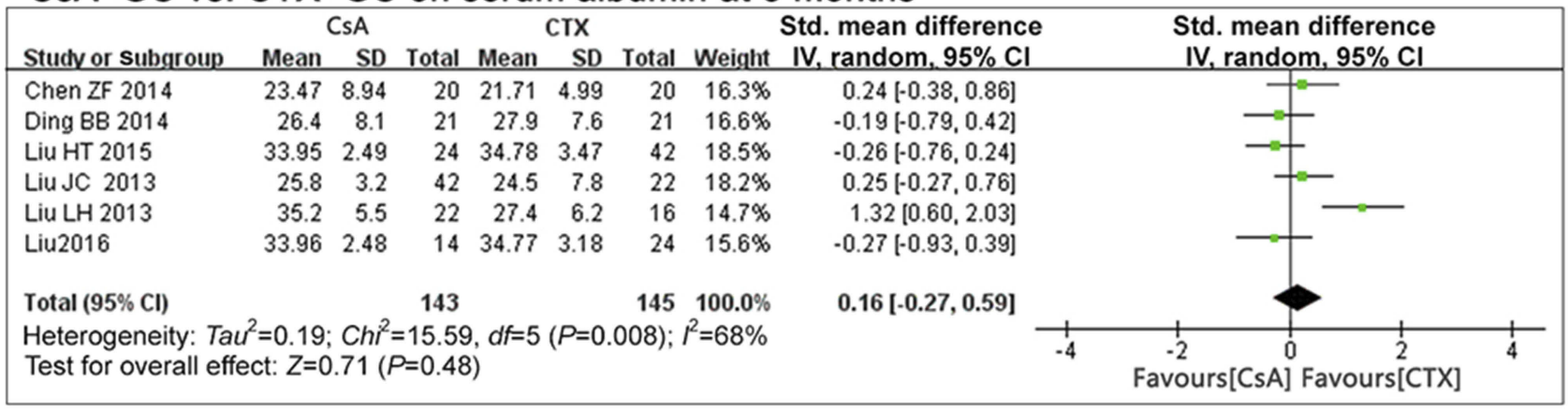

$\mathrm{CsA}+\mathrm{GC}$ vs. $\mathrm{CTX}+\mathrm{GC}$ on serum albumin at 6 months

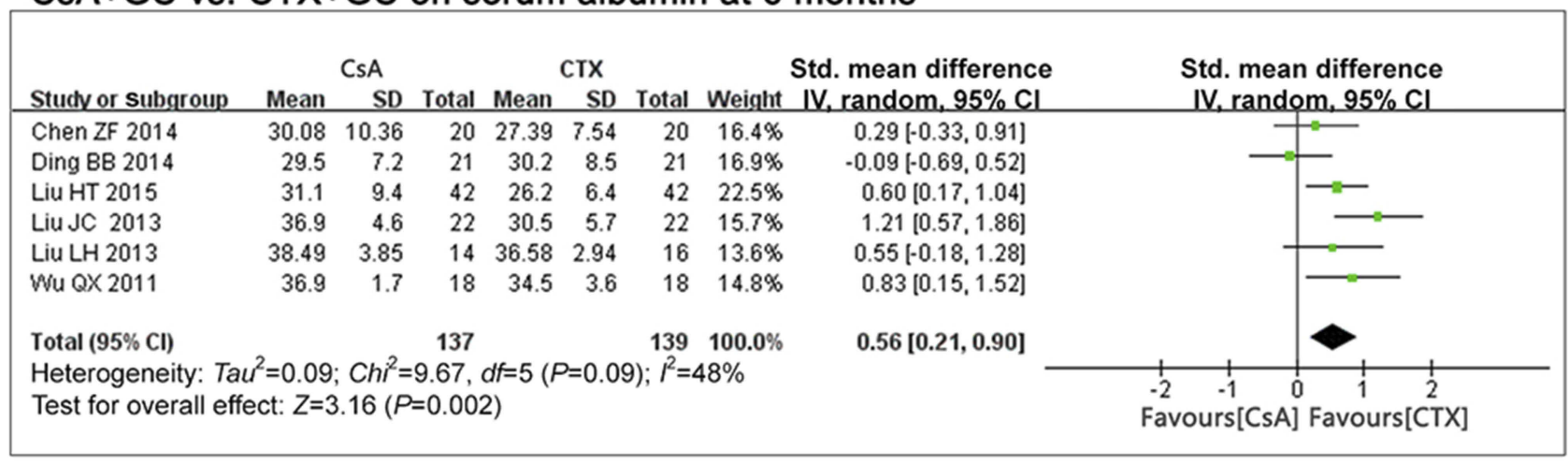

CsA+GC vs. CTX+GC on serum albumin at 12 months

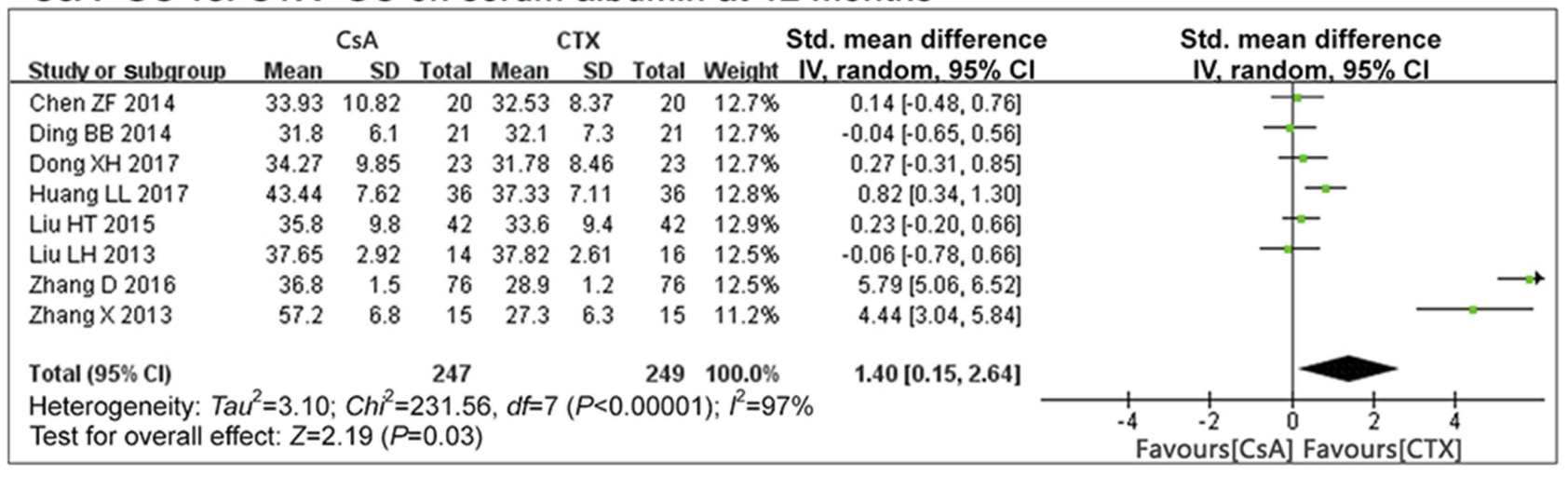

Figure 4 The effect of $C s A+G C$ vs $C T X+G C$ on serum albumin in patients with IMN in Asian populations.

Abbreviations: CsA, cyclosporine A; CTX, cyclophosphamide; GC, glucocorticoid; IMN, idiopathic membranous nephropathy.

$\mathrm{MD}=0.02,95 \% \mathrm{CI}:-0.28$ to 0.33 ; Table 2$),{ }^{17,19,28} 6$ months $^{17,19,28}(P=0.73, \mathrm{MD}=-0.05,95 \% \mathrm{CI}:-0.25$ to 0.36 ; Table 2) or 12 months $(P=1.00, \mathrm{MD}=-0.00,95 \%$ CI: -0.27 to 0.27 ; Table 2). ${ }^{17,19,20,28}$

However, four studies ${ }^{17,20,32,34}$ indicated that white blood cell levels were lower with CTX treatment $(P<0.00001, \mathrm{MD}=1.31,95 \% \mathrm{CI}: 1.04-1.58$; Table 2$)$ at 12 months.

Two studies ${ }^{21,30}$ reported no difference in urea nitrogen $(P=0.99, \mathrm{MD}=-0.00,95 \% \mathrm{CI}:-0.38$ to 0.38 ; Table 2).

\section{Adverse events}

In comparing $\mathrm{CsA}+\mathrm{GC}$ vs $\mathrm{CTX}+\mathrm{GC}$, the CsA group had more hirsutism ${ }^{17,19,22,27,29,30,34}(P=0.0001$, $\mathrm{OR}=9.28, \quad 95 \% \mathrm{CI}=3.00, \quad 28.69 ; \quad$ Table 3$), \quad$ gingival hyperplasia ${ }^{17-19,22,25-27,30,34} \quad(P=0.01, \quad \mathrm{OR}=3.96, \quad 95 \%$ $\mathrm{CI}=1.37$, 11.43; Table 3), elevated uric acid ${ }^{17,18,20,22,26,27}$ $(P=0.003, \mathrm{OR}=5.59,95 \% \mathrm{CI}: 1.79,17.41 ; \quad$ Table 3$), \quad$ and higher blood pressure ${ }^{17,18,20,22,25-27,29} \quad(P=0.0002$, $\mathrm{OR}=7.10,95 \% \mathrm{CI}: 2.50,20.14$; Table 3).

Gastrointestinal syndrome ${ }^{19,22,25-27,30} \quad(P<0.00001$, $\mathrm{OR}=0.05, \quad 95 \% \mathrm{CI}=0.02,0.17 ; \quad$ Table 3$), \quad$ hemorrhagic 
cystitis $^{18,22,30}(P=0.02, \mathrm{OR}=0.13,95 \% \mathrm{CI}=0.02,0.74$; Table $3)$, hepatic lesion ${ }^{17,19,20,25-27,29,30,34}$ ( $P=0.002, \mathrm{OR}=0.27$, $95 \% \mathrm{CI}=0.12,0.61$; Table 3), leucopenia ${ }^{17-20,22,26,27,30,34}$ $(P<0.00001, \mathrm{OR}=0.10,95 \% \mathrm{CI}=0.04,0.28$; Table 3$)$ as well as alopecia $^{17,18,22}(P=0.02, \quad \mathrm{OR}=0.12,95 \% \mathrm{CI}=0.02,0.67$; Table 3 ) were more frequent in the CTX group.

There were no differences in tremor ${ }^{19,22,27,30}(P=0.32$, $\mathrm{OR}=1.97, \quad 95 \% \quad \mathrm{CI}=0.52,7.41 ; \quad$ Table 3$)$ or serum creatinine $^{25-27,29}(P=0.06, \mathrm{OR}=4.43,95 \% \mathrm{CI}=0.91,21.43$; Table 3).

\section{Discussion}

In the current meta-analysis, comparison of the CsA vs the CTX, even though CsA had more rapid effects on CR compared to CTX at 3 months, there were no differences in $\mathrm{CR}$ at 6 months or 12 months. While CsA had lower inefficacy rates and a higher TR rate than the CTX group across the total treatment period (3 months, 6 months, and 12 months), it also had a higher relapse rate. There were no differences in $\mathrm{CR}, \mathrm{NR}$, or $\mathrm{TR}$ rates between $\mathrm{CsA}$ and MMF. As for the CsA compared to TAC, TAC had significantly higher $\mathrm{CR}$ and TR rates, and decreased NR.

The differences in the clinical index of the CsA group vs the CTX group are as follows: CsA has greater efficacy in lowering proteinuria levels only at 12 months, not at 3 or 6 months, Similar patterns were observed with increased serum protein levels and decreased serum cholesterol levels, which only differed in the later periods (6 and 12 months) but not at 3 months. White blood cells count at 12 months with CTX was significantly lower. CsA tended to be more effective in decreasing serum triacylglycerol. There were no differences in other clinical indicators, such as serum creatinine levels and the ALT, and urea nitrogen.

In comparing $\mathrm{CsA}+\mathrm{GC}$ vs $\mathrm{TAC}+\mathrm{GC}$, TAC improved serum protein levels as well as reduced serum triacylglycerol compared to CsA. There were no differences in proteinuria, serum creatinine, or the serum cholesterol levels.

Adverse event rates also differed between CsA and CTX treated groups. Leucopenia, hemorrhagic cystitis, and alopecia were only observed in the CTX group. Gingival hyperplasia, hirsutism, elevated blood pressure, and elevated blood creatinine were only reported with CsA treatment. All of these adverse events manifested apparent diversity, except the elevated blood creatinine. Rates of other adverse events, including blood creatinine and tremor, were not significantly different. However, gastrointestinal syndrome, liver function lesion, was observed more often in the CTX group. Elevated uric acid levels were more common with CsA. To conclude, patients treated with CTX were more likely to have severe side effects compared to the CsA group.

Overall, CsA had better efficacy than CTX, with milder adverse effects but a higher relapse rate with short-term treatment. There were no significant differences between CsA and MMF in NR, CR, or TR rates. Although the TAC group had higher TR and $\mathrm{CR}$, the NR rate was less than the CsA group.

Previous, meta-analyses have assessed the effects of CsA in treating IMN. Qiu et al, ${ }^{39}$ calculated the TR and observed that the CsA took effect more quickly than CTX, but this was confined to the first 6 months of treatment in IMN patients (eight studies). However, there were no increased effects of CsA on TR rate (six studies), serum

Table 3 Meta-analysis of the safety of CsA in induction therapy of patients with IMN (CsA vs CTX)

\begin{tabular}{|l|l|l|l|l|l|}
\hline \multirow{2}{*}{ Indicators } & Studies & Q test & Q test & OR \\
\cline { 2 - 6 } & Number & P-value & P-value & $\mathbf{( 9 5 \% C l )}$ \\
\hline Hirsutism & 7 & 0.97 & Fixed & $9.28(3.00,28.69)$ & 0.0001 \\
Alopecia & 3 & 0.81 & Fixed & $0.12(0.02,0.67)$ & 0.02 \\
Gingival hyperplasia; & 9 & 1.00 & Fixed & $3.96(1.37,11.43)$ & 0.01 \\
Hemorrhagic cystitis & 3 & 0.83 & Fixed & $0.13(0.02,0.74)$ & 0.02 \\
Liver function lesion & 9 & 0.73 & Fixed & $0.27(0.12,0.61)$ & 0.002 \\
Elevated serum creatinine & 4 & 0.97 & Fixed & $4.43(0.91,21.43)$ & 0.06 \\
Elevated blood pressure & 8 & 0.88 & Fixed & $7.10(2.50,20.14)$ & 0.0002 \\
Elevated uric acid & 6 & 0.62 & Fixed & $5.59(1.79,17.41)$ & 0.003 \\
Tremor & 4 & 0.52 & Fixed & $1.97(0.52,7.41)$ & 0.32 \\
Gastrointestinal syndrome & 6 & 0.93 & Fixed & $0.05(0.02,0.17)$ \\
Leucopenia & 9 & 0.96 & Fixed & $0.10(0.04,0.28)$ \\
\hline
\end{tabular}

Abbreviations: CsA, cyclosporine A; CTX, cyclophosphamide; IMN, idiopathic membranous nephropathy. 
albumin or proteinuria at 12 months. Ren et al, ${ }^{40}$ also performed a meta-analysis and concluded that CsA (six RCTs) was not superior to CTX in therapeutic effects (including CR and PR rates), when compared with nonimmunosuppressive treatment. Xie et $\mathrm{al}^{41}$ reported that CsA had no effect improvement in CR rate, but better responsiveness by pooling two included studies (116 patients). In the current meta-analysis, we assessed 16 RCTs (1,094 patients) and found that CsA had lower inefficacy rates and higher TR rates when compared with CTX. CsA also had enhanced long-term efficacy in elevating serum protein levels and lowering proteinuria levels (at 12 months), similar to studies of Ren et al. ${ }^{40}$ Qiu et al, ${ }^{39}$ observed that CsA patients were more likely to relapse in comparison with CTX or TAC, and our study reported a higher relapse rate with $\mathrm{CsA}+\mathrm{GC}$ compared to $\mathrm{CTX}+\mathrm{GC}$. Qiu et $\mathrm{al}^{39}$ also indicated a tendency for differences in adverse events (more severe adverse events like leukopenia, alopecia and liver damage with CTX, other events like hirsutism, gingival hyperplasia, worsening hypertension, and hyperuricemia occurred more often with CsA).

Compared with the above meta-analyses, our metaanalysis included a greater number of RCTs to assess the safety and efficacy of CsA, which enhanced the strength of our conclusions. This meta-analysis was the first to compare TAC with CsA in patients with IMN. We carefully assessed longitudinal effects, and provided greater details on adverse events.

However, there was some limitations in this meta-analysis. The quality of some of the included studies was low, and some sample sizes were small. The sample sizes of some specific comparisons for assessing adverse events were small, making it difficult to detect differences.

CsA has recently attracted the attention of a wide range of researchers', and results of clinical trials of CsA suggest that it could replace CTX as the primary medication for treating IMN. Our study summarized the existing randomized clinical trial data and calculated whether CsA performed better than CTX, TAC, and MMF in IMN in Asian populations. In conclusion, CsA group has the potential to replace CTX, but nephrotoxicity should be carefully considered. Additional studies of the efficacy and safety of other medications such as MMF, TAC, and AZA are needed.

\section{Acknowledgments}

This study was supported by Guangzhou Medical Key Discipline Construction Project (2017-2019), the Natural
Science Foundation of the Guangdong Province (no. 2015A030310386), and Guangdong Medical Science and Technology Research Fund Project (no. A2018336). Shujun Lin and Hong-Yan Li are co-first authors for this study.

\section{Disclosure}

The authors report no conflicts of interest in this work.

\section{References}

1. Zeng C, Chen H, Wang R, et al. Etiology and clinical characteristics of membranous nephropathy in Chinese patients. Am J Kidney Dis. 2008;52(4):691-698. doi:10.1053/j.ajkd.2008.06.006

2. Alfaadhel T, Cattran D. Management of membranous nephropathy in Western countries. Kidney Dis (Basel). 2015;1(2):126-137. doi: $10.1159 / 000437287$

3. Polanco N, Gutierrez E, Covarsi A, et al. Spontaneous remission of nephrotic syndrome in idiopathic membranous nephropathy. $J \mathrm{Am}$ Soc Nephrol. 2010;21(4):697-704. doi:10.1681/ASN.2009080861

4. Quaglia M, Stratta P. Idiopathic membranous nephropathy: management strategies. Drugs. 2009;69(10):1303-1317. doi:10.2165/ 00003495-200969100-00002

5. Daniel C. Management of membranous nephropathy: when and what for treatment. $J$ Am Soc Nephrol. 2005;16(5):1188-1194. doi:10.1681/ASN.2005010028

6. Glassock RJ. The treatment of idiopathic membranous nephropathy: a dilemma or a conundrum? Am J Kidney Dis. 2004;44(3):562-566.

7. Jha V, Ganguli A, Saha TK, et al. A randomized, controlled trial of steroids and cyclophosphamide in adults with nephrotic syndrome caused by idiopathic membranous nephropathy. J Am Soc Nephrol. 2007;18(6):1899-1904. doi:10.1681/ASN.2007020166

8. Ruggenenti P, Remuzzi G. Idiopathic membranous nephropathy: back to the future? Lancet. 2013;381(9868):706-708. doi:10.1016/S01406736(12)62033-9

9. Waldman M, Austin HR. Treatment of idiopathic membranous nephropathy. $J \quad A m$ Soc Nephrol. 2012;23(10):1617-1630. doi:10.1681/ASN.2012010058

10. Zhu L, Liu L, Yao L, et al. Efficacy and safety of tacrolimus versus cyclophosphamide for primary membranous nephropathy: a meta-analysis. Drugs. 2017;77(2):187-199. doi:10.1007/s40265-016-0683-z

11. van Den Brand JAJG, Ruggenenti P, Chianca A, et al. Safety of rituximab compared with steroids and cyclophosphamide for idiopathic membranous nephropathy. J Am Soc Nephrol. 2017;28 (9):2729. doi:10.1681/ASN.2016080886

12. Goumenos DS. What have we learned from the use of ciclosporin A in the treatment of nephrotic patients with idiopathic membranous nephropathy? Expert Opin Pharmacother. 2008;9(10):1695-1704. doi:10.1517/14656566.9.10.1695

13. Radhakrishnan J, Halevy D. Cyclosporin treatment of glomerular diseases. Expert Opin Investig Drugs. 2000;9(5):1053-1063. doi:10.1517/13543784.9.5.1053

14. Kshirsagar AV, Nachman PH, Falk RJ. Alternative therapies and future intervention for treatment of membranous nephropathy. Semin Nephrol. 2003;23(4):362-372.

15. Ponticelli C. Cyclosporine: from renal transplantation to autoimmune diseases. Ann N Y Acad Sci. 2005;1051:551-558.

16. Naesens M, Kuypers DR, Sarwal M. Calcineurin inhibitor nephrotoxicity. Clin J Am Soc Nephrol. 2009;4(2):481-508.

17. Chen ZF, Zhang JW, Xia NN, et al. Comparison on the therapeutic effect of cyclosporine A and cyclophosphamide in the treatment of idiopathic membranous nephropathy. China Med Pharm. 2014;4(16):16-18. 
18. Deng SS, Zhu MZ, Bo M. Clinical observation of cyclosporine A versus cyclophosphamide in the treatment of idiopathic membranous nephropathy. Chin J Mod Drug Appl. 2018;12(14):125-127.

19. Ding BB. Comparison of the effects of two therapeutic regimen in the treatment of idiopathic membranous nephropathy. Chin J Prim Med Pharm. 2014;21(21):3238-3241.

20. Dong XH, Miu JL, Chen HL. The comparison of the clinical effect of cyclosporine A versus cyclophosphamide in the treatment of idiopathic membranous nephropathy. PJCCPVD. 2017;25(S1):80-81.

21. Huang LL. The observation of long-term efficacy and safety of cyclosporine A combined with glucocorticoid in the treatment of idiopathic membranous nephropathy. Hebei Med. 2017;23(07):1202-1206.

22. Huang ZM, Chen KJ. The observation of the efficacy, relapse rate and the side effect of cyclosporine A versus cyclophosphamide in the treatment of idiopathic membranous nephropathy. J North Pharm. 2016;13(9):66.

23. Xie J, Guo W, Zhang QD, et al. Clinical effect of cyclophosphamide or tacrolimus combined with glucocorticoids in the treatment of idiopathic membranous nephropathy. Chin J Integr Tradit West Nephrol. 2014;15(08):716-718.

24. Li LL. Clinical comparison of the hormone combined with different immunosuppressive agents in the treatment of idiopathic membranous nephropathy. Guide China Med. 2016;14(16):180-181.

25. Liu AQ, Liu GP, Yu L, et al. Clinical observation of cyclosporine A in the treatment of idiopathic membranous nephropathy. Inner Mongolia Med J. 2016;48(12):1505-1506.

26. Liu JC, An ZM, Zhang LC, et al. Efficacy of cyclosporine A combined with glucocorticoid in treatment of membranous nephropathy. $J$ Qiqihar Univ Med. 2013;34(13):1910-1912.

27. Liu LH, Ma SY, Liu ZY, et al. The observation of efficacy of cyclosporine $\mathrm{A}$ in the treatment of idiopathic membranous nephropathy. Anhui Med J. 2013;34(8):1217-1219.

28. Liu HT. The observation of efficacy of cyclosporine A combined with glucocorticoid in the treatment of idiopathic membranous nephropathy. Chin J Prim Med Pharm. 2015;22(16):2467-2470.

29. Sa RL. The analysis of safety and efficacy of cyclosporine A in the treatment of idiopathic membranous nephropathy. China Health Care Nutr. 2018;28(1):325-326.

30. Wu QX, Gong ZF. Clinical observation of 20 cases of membranous nephropathy treated with low and medium dose cyclosporine. China Pharm. 2011;14(1):115-117.
31. Yang J, Liu Y, Zhang Y, et al. Glucocorticoid and immunosuppressive therapy with high risk factors for idiopathic membranous nephropathy in 46 cases. Prog Mod Biomed. 2013;13(14):2710-2712.

32. Zhang D. The observation of clinical efficacy of cyclosporine A versus cyclophosphamide in treatment of membranous nephropathy. Health Med Res Prac. 2016;13(04):50-51.

33. Zhang M. Clinical observation of cyclophosphamide combined with glucocorticoid on membranous nephropathy. Chin J Biochem Pharm. 2016;36(1):121-123.

34. Zhang X. Low-dose of cyclosporine and low-dose glucocorticoid in the treatment of idiopathic membranous nephropathy. Guide China Med. 2013;11(30):121-122.

35. Choi J, Kim DK, Kim Y, et al. The effect of mycophenolate mofetil versus cyclosporine as combination therapy with low dose corticosteroids in high-risk patients with idiopathic membranous nephropathy: a multicenter randomized trial. J Korean Med Sci. 2018;33(9): e74.

36. Guo Y. The observation of efficacy of the tacrolimus in the treatment of idiopathic membranous nephropathy. J North Pharm. 2016;13 (9):61.

37. Hu QF The Clinical Observation of the cyclosporine A Versus Tacrolimus Combined with Half-Dose of Glucocorticoid in the Treatment of Idiopathic Membranous Nephropathy [doctoral dissertation]. Xinxiang: Xinxiang Medical University; 2016.

38. Li QH The Clinical Observation of the cyclosporine A Versus Tacrolimus Combined with Half-Dose of Glucocorticoid in the Treatment of Idiopathic Membranous Nephropathy [doctoral dissertation]. Zhengzhou: Zhengzhou University; 2017.

39. Qiu TT, Zhang C, Zhao HW, et al. Calcineurin inhibitors versus cyclophosphamide for idiopathic membranous nephropathy: a systematic review and meta-analysis of 21 clinical trials. Autoimmun Rev. 2017;16(2):136-145.

40. Ren S, Wang Y, Xian L, et al. Comparative effectiveness and tolerance of immunosuppressive treatments for idiopathic membranous nephropathy: a network meta-analysis. PLoS One. 2017;12(9): e184398.

41. Xie G, Xu J, Ye C, et al. Immunosuppressive treatment for nephrotic idiopathic membranous nephropathy: a meta-analysis based on Chinese adults. PLoS One. 2012;7(9):e44330.

\section{Publish your work in this journal}

Drug Design, Development and Therapy is an international, peerreviewed open-access journal that spans the spectrum of drug design and development through to clinical applications. Clinical outcomes, patient safety, and programs for the development and effective, safe, and sustained use of medicines are a feature of the journal, which has also been accepted for indexing on PubMed Central. The manuscript management system is completely online and includes a very quick and fair peer-review system, which is all easy to use. Visit http://www. dovepress.com/testimonials.php to read real quotes from published authors. 